Accepted for publication by The Astronomical Journal - July 1, 2008.

Preprint typeset using $\mathrm{LAT}_{\mathrm{E} X} \mathrm{X}$ style emulateapj v. 11/26/04

\title{
THE NUCLEUS OF THE SAGITTARIUS DSPH GALAXY AND M54: A WINDOW ON THE PROCESS OF GALAXY NUCLEATION.
}

\author{
M. Bellazzini \\ INAF - Osservatorio Astronomico di Bologna, via Ranzani 1, 40127, Bologna, Italy
}

R.A. IBATA

Observatoire Astronomique, Université de Strasbourg, CNRS, 11, rue de l'Université, F-67000 Strasbourg, France

\author{
S.C. Chapman ${ }^{2}$ \\ Institute of Astronomy, Madingley Road, Cambridge CB3 0HA
}

A.D. MACKEY

Institute for Astronomy, University of Edinburgh, Royal Observatory, Blackford Hill, Edinburgh EH9 3HJ

L. Monaco

European Southern Observatory, Alonso de Cordova 3107, Casilla 19001, Santiago, Chile

M.J. IRWIN

Institute of Astronomy, Madingley Road, Cambridge CB3 0HA

N.F. MARTIN

Max-Planck-Institut für Astronomie, Königstuhl 17, D-69117 Heidelberg, Germany

G.F. LEWIS

Institute of Astronomy, School of Physics, A29 University of Sydney, NSW 2006, Australia

AND

E. DALESSANDRO ${ }^{2}$

Dipartimento di Astronomia, Università di Bologna, via Ranzani 1, 40127, Bologna, Italy

Accepted for publication by The Astronomical Journal - July 1, 2008.

\begin{abstract}
We present the results of a thorough study of the nucleus of the Sgr dwarf spheroidal galaxy (Sgr dSph) and of the bright globular cluster M54 (NGC 6715) that resides within the same nucleus $(\mathrm{Sgr}, \mathrm{N})$. We have obtained accurate radial velocities and metallicity estimates for 1152 candidate Red Giant Branch stars of Sgr and M54 lying within 9' from the center of the galaxy, from Keck/DEIMOS and VLT/FLAMES spectra of the infrared Calcium II triplet. Using both velocity and metallicity information we selected two samples of 425 and 321 very-likely members of M54 and of Sgr,N, respectively. The two considered systems display significantly different velocity dispersion profiles: M54 has a steeply decreasing profile from $r=0^{\prime}$, where $\sigma \simeq 14.2 \mathrm{~km} / \mathrm{s}$, to $r \simeq 3.5$ where it reaches $\sigma \simeq 5.3 \mathrm{~km} / \mathrm{s}$, then it appears to rise again to $\sigma \simeq 10 \mathrm{~km} / \mathrm{s}$ at $r \sim 7^{\prime}$. In contrast Sgr,N has a uniformly flat profile at $\sigma \simeq 9.6 \mathrm{~km} / \mathrm{s}$ over the whole $0^{\prime} \leq r \leq 9^{\prime}$ range. Using data from the literature we show that the velocity dispersion of Sgr remains constant at least out to $r \sim 100^{\prime}$ and there is no sign of the transition between the outer flat-luminosity-profile core and the inner nucleus in the velocity profile. These results - together with a re-analysis of the Surface Brightness profile of $\mathrm{Sgr}, \mathrm{N}$ and a suite of dedicated N-body simulations - provide very strong support for the hypothesis that the nucleus of Sgr formed independently of M54, which probably plunged to its present position, coincident with Sgr,N, because of significant decay of the original orbit due to dynamical friction.

Subject headings: galaxies: dwarf - globular clusters: individual(NGC 6715) — stars: kinematics galaxies: nuclei — galaxies: individual (Sgr dSph)
\end{abstract}

Electronic address: michele.bellazzini@oabo.inaf.it

Electronic address: ibata@astro.u-strasbg.fr

${ }^{1}$ University of Victoria, Victoria, BC, V8W 3P6, Canada

Electronic address: schapman@ast.cam.ac.uk

Electronic address: dmy@roe.ac.uk
Electronic address: lmonaco@eso.org

Electronic address: mike@ast.cam.ac.uk

Electronic address: martin@mpia-hd.mpg.de

Electronic address: gH@physics.usyd.edu.au

2 ASI, Centro di Geodesia Spaziale, contrada Terlecchia, I-75100, 


\section{INTRODUCTION}

The occurrence of stellar nuclei at the photometric center of several dwarf elliptical galaxies has been the subject of statistical investigations since the pioneering study by Binggeli, Sandage \& Tammann (1985) and Binggeli. Tammann \& Sandage (1987), and nucleated dwarf ellipticals (dE,N) have become a generally recognized and well studied class of galaxies in their own right (see Gallagher \& Wyse 1994; Ferguson \& Binggeli 1994, and references therein). In the last few years the results from systematic studies performed with the instrumentation on board of the Hubble Space Telescope (HST), has revolutionized the field, providing strong evidence supporting the possibility of an intimate connection between the process of nucleation and the process of galaxy formation as a whole. In particular:

- It was generally accepted that stellar nuclei occurred in a significant but minor fraction of dwarf elliptical galaxies $(\sim 25 \%$ Binggeli. Sandage \& Tammann 1985); on the other hand, the high resolution HST analysis of a large and well selected sample of Virgo dEs by Côté et al. (2006, hereafter C06) has revealed that the fraction of $\mathrm{dE}, \mathrm{Ns}\left(f_{n}\right)$ can be as high as $66 \% \leq f_{n} \leq 87 \%$. Strong support to a significant upward revision of $f_{n}$ comes also from the recent ground-based study by Grant, Kuipers, \& Phillipps (2005). Hence, nucleation appears as the natural status of dEs instead of an exceptional occurrence (see C06 for details and discussion).

- An HST study of a large sample of Sa-Sd galaxies by Böker et al. (2004) found that a similarly high fraction of stellar nuclei is found also among these late type spirals, $f_{n} \simeq 77 \%$. In general, the most recent studies agree in finding a fraction of nucleation larger than $50 \%$ in any kind of galaxy (see, for example Carollo, Stiavelli \& Mack 1998; Balcells. Graham \& Peletier 2007; Ferrarese \& Côté 2006; Böker 2007, and references therein), with the only exception of those brighter than $M_{B} \simeq-20.3 \mathrm{mag}$ (Ferrarese \& Côté 2006, hereafter FC06). Hence, nucleation seems to occur very frequently in both dwarf and giant galaxies (see Graham \& Guzmán 2003, and references therein, for a deeper discussion of the dwarf/giant dichotomy).

- Stellar galactic nuclei are found to obey the same scaling relation that links Supermassive Black Holes $(\mathrm{SBH})$ with their host galaxies (Wehner \& Harris 2006, C06, FC06). It has been suggested that "...SBHs and stellar nuclei are nothing but complementary incarnation of Central Massive Objects - they likely share a common formation mechanism and follow a similar evolutionary path..." (FC06). In this view, the accumulation of a compact overdensity of baryons at the very center would be a common process during galaxy

Matera, Italy

Electronic address: emanuele.dalessandr2@unibo.it formation, while its further evolution into a SBH or a stellar nucleus would depend on various "local" circumstances.

- The similarity between stellar nuclei, the brightest globular clusters and the recently discovered Ultra Compact Dwarf (UCD) galaxies (Drinkwater et al. 2003) clearly suggests possible links among these classes of stellar systems (Freeman 1993; Lotz et al. 2001; Lotz, Miller \& Ferguson 2004; Bassino, Muzzio \& Rabolli 1994; Hasegan et al. 2005; Federici et al. 2007; Böker 2007). In particular it has been suggested either that nuclei can form by merging of pre-existing globular clusters (Lotz et al. 2001, and references therein), or that some objects currently classified as globular clusters may be in fact galactic nuclei, the only relics of the tidal shredding of their host galaxies that once orbited the Milky Way and/or M31 (see Mackev \& van den Bergh 2005; Federici et al. 2007; Brodie \& Strader 2006, and references therein), or even that most/all globulars were nuclei of Galactic building blocks (i.e. dwarf galaxies, see Freeman 1993; Freeman \& Bland-Hawthorn 2002; Böker 2007, BS, and references therein).

Within the scenario briefly outlined above, the crucial relevance of any independent observational fact that may provide some insight on the process of nucleation is clearly manifest. The mechanisms for the formation of stellar nuclei that have been suggested until now belong to two broad classes: (a) the orbital decay and merging at the center of the parent galaxies of pre-existing stellar systems (star clusters), and (b) the accumulation of gas (of various origins) in the very center of a galaxy and its subsequent transformation into stars (see Grant, Kuipers, \& Phillipps 2005, for a detailed description of various proposed flavors of these classes of models).

At present, all the observational constraints we have in hand come from the study of the integrated properties of samples of distant galaxies/nuclei. The nearest galaxies that are known to host a stellar nucleus are M32, M33 and NGC205, in the Local Group. Even in the most favorable case of NGC205, the structure of the nucleus can be studied in some detail but it can be resolved into individual stars only partially, even with HST imaging (Valluri et al. 2005). In this context the case of the Sagittarius dwarf spheroidal galaxy (Sgr dSph, Ibata. Irwin \& Gilmore 1994) constitutes an absolute unicum (Monaco et al. 2005a, hereafter M05a).

The Sgr dSph is a dwarf satellite $\left(L \sim 2-5 \times 10^{7} L_{\odot}\right)$ of the Milky Way (Ibata et al. 1997) whose main body is at $D_{\odot}=26.3 \pm 1.8 \mathrm{kpc}$ from us and $R_{G C} \simeq 18.7 \mathrm{kpc}$ from the center of the Galaxy (Monaco et al. 2004). It is devoid of gas (Burton \& Lockman 1999) and its stellar content is dominated by an intermediate-age $(\sim 6-8 \mathrm{Gyr}$ old, see Bellazzini et al. 2006a, and references therein) metal-rich $\left(\langle Z\rangle \sim \frac{2}{5} Z_{\odot}\right.$, Monaco et al. 2005b, and references therein) population, while $\simeq 10 \%$ of old and metal poor stars are also present (Monaco et al. 2003). The Sgr dSph is currently being destroyed by the Galactic tidal field (Ibata et al. 1997). The tidally stripped stars form a huge and coherent tidal tail system that can be 
observed over the whole celestial sphere (Sgr Stream, see Ibata et al. 2001; Majewski et al. 2003, and references therein). There are four globular clusters within the main body of the galaxy (Ibata. Irwin \& Gilmore 1994; Da Costa \& Armandroff 1995; Montegriffo et al. 1998) and probably several others that have been stripped from the main body and are now associated with the Sgr Stream (Bellazzini. Ferraro \& Ibata 2003a; Bellazzini et al. 2003b; Carraro, Zinn \& Moni Bidin 2007).

Particularly noticeable, however, is the very bright $\left(M_{V}=-10.0\right.$, Harris 1996$)$ metal-poor $([\mathrm{Fe} / \mathrm{H}]=$ -1.55) (Brown. Wallerstein \& Gonzalez 1999) globular cluster M54 (NGC6715), located exactly at the center of the light distribution of the Sgr galaxy. For this reason, immediately after the discovery of the galaxy it was suggested that M54 could be the actual nucleus of Sgr dSph (Bassino \& Muzzio 1995; Sarajedini \& Lavden 1995). Some doubt on this hypothesis was advanced by Da Costa \& Armandroff (1995) based on the circumstance that the integrated color of M54 is much bluer than that of its host galaxy, while the opposite was generally believed to occur in $\mathrm{dE}, \mathrm{Ns}$; however Sarajedini \& Layden (1997) noticed that there were exceptions to this rule. In fact, the latest studies suggest that the opposite may be true (Lotz, Miller \& Ferguson 2004, see also C06). Since the mean metallicity of M54 and of the population dominating the Sgr galaxy differ by one full dex, their Red Giant Branch (RGB) stars (and in some cases also their Horizontal Branch stars, HB) can be easily discriminated from each other in a Color Magnitude Diagram (CMD); at the same magnitude the RGB of Sgr is much redder than that of M54. Selecting genuine Sgr stars in this way Layden \& Sarajedini (2000) and Majewski et al. (2003) independently found an overdensity of Sgr stars that appear concentric with M54 and have a similar spatial scale. Using the same technique and a very large optical photometry database M05a demonstrated that Sgr has actually a nucleus of metal rich stars (in the following we will refer to this structure as the nucleus of the Sagittarius galaxy, $S g r, N$ for brevity, if not otherwise stated). This stellar structure has the same center as M54, to within the uncertainties, but it displays a different surface brightness profile, suggesting a different origin from the cluster. The strong incompleteness in the innermost $\sim 10^{\prime \prime}$ prevented M05a from obtaining accurate estimates of the structural parameters of the Sgr nucleus: they derived $-10.0 \leq M_{V} \leq-7.6$, $\mu_{V}(0) \lesssim 18.5 \mathrm{mag} / \operatorname{arcsec}^{2}$, and, with a tentative fit of a King (1966) model $r_{c} \lesssim 0.21^{\prime}$ and $r_{t} \sim 17^{\prime}$ (where $\mathrm{C}=\log \left(r_{t} / r_{c}\right) \sim 1.9$; we will refine these parameters in Sect. 2, below). They also found that the observed properties of the nucleus of the Sgr galaxy were fully compatible with those of known nuclei of dwarf ellipticals. Moreover, the recent study by Siegel et al. (2007) has shown that while the stellar budget of $\mathrm{Sgr}, \mathrm{N}$ is dominated by the same intermediate-age population found in the surrounding galaxy, there is clear evidence of more recent and repeated episodes of star formation (not observed in extra-nuclear fields, Bellazzini et al. 2006a), pointing to a history of subsequent phases of re-accumulation of gas followed by star formation bursts, very similar to what is observed by Rossa et al. (2006) in a large sample of distant galactic nuclei.
In any case, the key result of M05a is that Sgr is a nucleated galaxy independently of the presence of M54; even if one were able to remove the cluster from the galaxy by magic, a nucleus made of typical Sgr stars would still be there. To explain the strict spatial coincidence of M54 and the nucleus, M05a proposed two possibilities: (a) the nucleus formed in situ from processed Sgr gas that has fallen to the bottom of the galactic potential well, and M54 was (independently) driven into the same place by dynamical friction; (b) M54 was born in the present place (or was driven there as in case a) and formed a co-spatial overdensity of Sgr stars by capturing them (or the gas from which they formed) from the surroundings. The key difference between the two hypothesis can be summarized by the questions "did M54 provide the mass seed around which the metal-rich nucleus of Sgr was assembled? is M54 the main contributor of the mass budget in the innermost $\sim 100$ pc of the Sgr galaxy?", that enclose the basic point of hypothesis (b).

Hypothesis (a) was found to be fully compatible with all the available information, while it was not possible to consider case (b) in more depth. In a recent paper, Mieske \& Baumgardt (2007) studied in detail the process of the capture of "field" stars from an intervening star cluster and they concluded that, even in the most favourable cases, the fraction of stars captured in a Hubble time is negligible, less that $10^{-4}$ of the cluster mass. The range of conditions studied by Mieske \& Baumgardt (2007) includes the "M54 within Sgr" case, hence their conclusions are fully applicable here. Nevertheless, the results by Mieske \& Baumgardt (2007) do not exclude the possibility that M54 operates as a collector of processed gas at the center of Sgr.

However it seems quite reasonable to expect that if case (b) is true, the stars of the Sgr,N structure should share the same kinematics as M54 stars, or, at least, their kinematics should be compatible with a mass-followslight model, or, finally, their kinematics should be different from that of extra-nuclear Sgr stars, as they would be bound to M54 and would orbit within its potential (Gilmore et al. 2007). Note that M54 and Sgr,N are enclosed within the innermost $10^{\prime}$ of a galaxy whose core radius and limiting radius are as large as $r_{c}=224^{\prime} \pm 10^{\prime}$ and $r_{t}=1801^{\prime} \pm 112^{\prime}$, respectively (Ma jewski et al. 2003). Hence, even if the main body of Sgr is clearly undergoing tidal disruption the kinematics of the nuclear region should be unaffected and is consequently expected to provide a trustworthy insight on the inner mass distribution (Muñoz, Majewski \& Johnston 2007).

To follow-up these ideas and to obtain a deeper insight into this unique case of a galactic nucleus that can be studied in vivo on a star by star basis, we performed an extensive spectroscopic survey, principally aimed at the study of the kinematics of Sgr,N and M54. Here we present the main results of this survey. The plan of the paper is as follows. As a preliminary step we re-analyse the Surface Brightness profile of Sgr,N with new data in Sect. 2. In Sect. 3 we describe the observation and data reduction of our spectroscopic survey, we test the reliability and the accuracy of our measures, the observed metallicity distribution is briefly discussed and, finally, the selection of the samples is described in detail. Sect. 4 is devoted to the kinematical analysis of the M54 and Sgr,N samples and in Sect. 5 we briefly present the results 

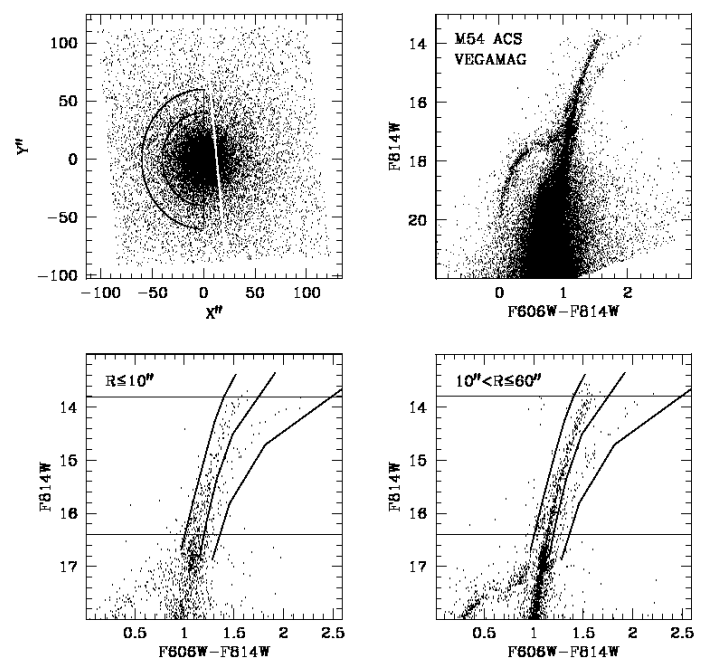

FIG. 1.- Upper left panel: the annuli and semi-annuli adopted for radial star counts are plotted on the X,Y map of the stars having $\mathrm{F} 814 \mathrm{~W}<20.0 ; \mathrm{X}, \mathrm{Y}$ are the local coordinates as defined in Sect. 3., below, but in arcseconds. Upper right panel: overall CMD; note the difference among the steep and star rich RGB of M54 and the redder and sparser RGB of Sgr, N, for F814W $<17.0$. Lower panels: the selection of M54 and Sgr,N RGB stars adopted as density tracers is illustrated for two radial ranges. The horizontal lines enclose the magnitude range of the selection, the bluest and the reddest red lines select the RGB stars of both systems and the middle red line separates the sample into M54 members (bluer than the line) and Sgr,N members (redder than the line).

of a suite of N-body simulations aimed at the study of the orbital decay of M54 within the Sgr galaxy. Finally, in Sect. 6 we summarize and discuss our results.

\section{REFINING THE STRUCTURAL PARAMETERS OF $\mathrm{SGR}, \mathrm{N}$}

To gain a better insight into the Surface Brightness (SB) profile of Sgr,N, we reduced archive data obtained with the Wide Field Channel (WFC) of the Advanced Camera for Survey (ACS) on board of the Hubble Space Telescope (HST). This is a set of short and long exposure $\mathrm{F} 814 \mathrm{~W}$ and $\mathrm{F} 606 \mathrm{~W}$ images of the center of M54, taken within the GO 10775 Treasury Program (Sarajedini et al. 2007). The relative and absolute photometry of individual stars has been obtained following the same steps described in Sollima et al. (2007) for data taken from the same survey. Further details and the overall analysis of these data will be presented elsewhere; an independent study of the stellar populations of M54 and Sgr, N from the same images has been recently presented by Siegel et al. (2007). In the present context, we limit ourselves to complementing the SB profile of Sgr,N obtained by M05a with a couple of points in the innermost $10^{\prime \prime}$, using the same methodology as M05a, and, as a consequence, to obtain stronger constraints on the structural parameters of Sgr,N.

Fig. 1 clearly shows that the new ACS photometry allows one to discriminate the RGBs of M54 and Sgr,N by color even in the innermost $10^{\prime \prime}$. Using the selection illustrated in Fig. 1 we were able to reliably measure the ratio of the density of tracer stars (RGB and AGB, in the present case) in the two samples in any given radial annulus $\rho_{S g r, N} / \rho_{M 54}$, as it scales as the ratio of the
TABLE 1

Surface Brightness profile of the innermost $60^{\prime \prime}$ of SGr

\begin{tabular}{ccccc}
\hline $\begin{array}{c}r_{i} \\
r_{f}\end{array}$ & $\begin{array}{c}r_{f} \\
{ }^{\prime \prime}\end{array}$ & $\begin{array}{c}\mathrm{r}_{m} \\
\prime \prime\end{array}$ & $\begin{array}{c}\mu_{V} \\
\mathrm{mag} / \operatorname{arcsec}^{2}\end{array}$ & $\begin{array}{c}\mathrm{e}_{\mu_{V}} \\
\mathrm{mag} / \operatorname{arcsec}^{2}\end{array}$ \\
\hline 0 & 5 & 3.01 & 16.1 & 0.3 \\
0 & 10 & 5.35 & 16.6 & 0.2 \\
10 & 20 & 14.30 & 18.3 & 0.3 \\
20 & 40 & 27.84 & 20.0 & 0.4 \\
40 & 60 & 49.78 & 21.7 & 0.6 \\
\hline
\end{tabular}

Note. $-r_{i}$ and $r_{f}$ are the limits of the bins, $r_{m}$ is the average radius of the sample.

TABLE 2

Parameters of the King model that Best fits the SGR,N PROFILE.

\begin{tabular}{cccc}
\hline Parameter & Estimate & Uncertainty & Units \\
\hline$r_{c}$ & 0.05 & \pm 0.01 & arcmin \\
$\mathrm{C}$ & 1.90 & $\ldots$ & $\ldots$ \\
$r_{t}$ & 4.00 & \pm 0.8 & arcmin \\
$r_{h}$ & 0.42 & \pm 0.08 & arcmin \\
$r_{l}$ & $\simeq 10.5$ & $\ldots$ & arcmin \\
$\mu_{V}(0)$ & 15.3 & \pm 0.2 & $\mathrm{mag} / \operatorname{arcsec}^{2}$ \\
$M_{V}$ & -7.8 & \pm 0.2 & $\mathrm{mag}$ \\
\hline
\end{tabular}

Note. $-r_{t}$ is the tidal radius of the same King model, while $r_{l}$ is the observed limiting radius, defined as the radius at which the observed profile of the nucleus appears to join the profile of the overall Sgr galaxy. All the reported values and uncertainties have been estimated by assuming the reported value of C. The reported values of the surface brigthness are not corrected for interstellar extinction. ${ }^{a} r_{h}$ is the half-mass radius of the best fitting King (1966) model: it can be considered equivalent to the half-light radius, in the present case. The half-light radius of the King (1962) best fitting the SB profile of Sgr, $\mathrm{N}$ is $r_{h}=0.23^{\prime}$.

number of stars per annulus $N_{S g r, N} / N_{M 54}$. To avoid the inter-chip gap we used semi-annuli for $R>10^{\prime \prime}$. The quantity $2.5 \log \left(N_{S g r, N} / N_{M 54}\right)$, in turn, scales as the difference in Surface Brightness between the two systems (see M05a for details, and Renzini \& Buzzoni 1986; Renzini \& Fusi Pecci 1988, for the basis of the underlying evolutionary flux theorem) ${ }^{3}$. No attempt was made to estimate the ellipticity of the system; as the system appears quite spherical at large radii (see M05a) we assumed spherical symmetry. The coordinates of the centers of M54 and Sgr,N, as derived from the $2 \sigma$ clipped average $\mathrm{X}$ and $\mathrm{Y}$ of stars selected by color as in Fig. 1, are found to coincide to within $<2^{\prime \prime}$, in excellent agreement with M05a. The effects of the radial variation of completeness on the density ratio should be practically null, as we selected the M54 and Sgr,N samples in the same (bright) magnitude range $(13.8 \leq F 814 W \leq 16.4)$. To convert the estimated SB differences into an absolute scale we adopted the bestfit model profile of M54 by Trager, King \& Diorgovski (1995) as a "zero-point", consistent with M05a. In practice, for each observed $\Delta \mu^{S g r-M 54}\left(R_{i}\right)$ we obtained the surface brightness of Sgr,N at radius $R_{i}, \mu_{V}^{S g r}\left(R_{i}\right)$, as $\mu_{V}^{S g r}\left(R_{i}\right)=\mu_{V}^{M 54}\left(R_{i}\right)+\Delta \mu^{S g r-M 54}\left(R_{i}\right)$. The derived

3 We used synthetic CMDs produced with the dedicated BASTI web interface (Cordier et al. 2007) to check that a population of age $=12 \mathrm{Gyr}$ and $[\mathrm{Fe} / \mathrm{H}]=-1.5$ (taken as a proxy for M54) and a population of age $=6 \mathrm{Gyr}$ and $[\mathrm{Fe} / \mathrm{H}]=-0.4$ (taken as a proxy for Sgr,N) having the same total $V$ luminosity, places (approximately) the same number of stars in the selection windows shown in Fig. 1 


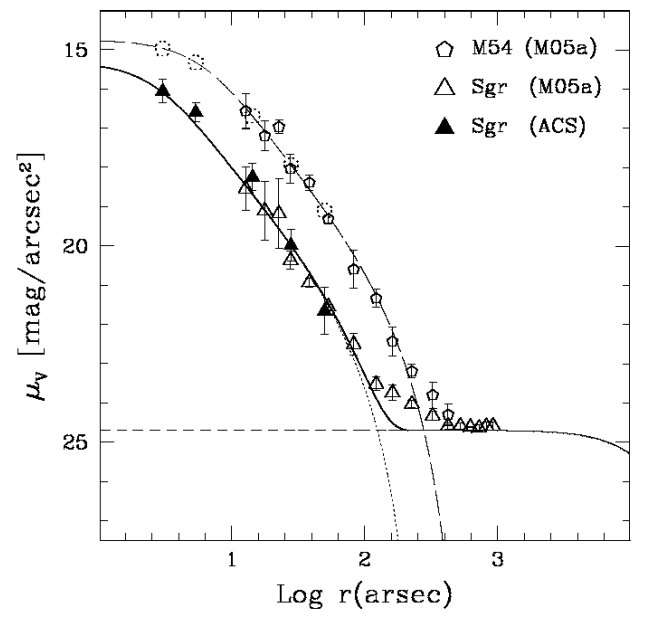

FIG. 2.- Surface brightness profiles of M54 (pentagons) and of Sgr (triangles). Continuous open symbols with errorbars are from M05a. The filled triangles have been obtained from the density ratio computed here from HST/ACS data and are normalized by adding them to the corresponding SB of the M54 best-fit model, here marked by dotted pentagons. The long dashed line is the bestfit King (1966) model found by Trager, King \& Diorgovski (1995) for M54; the short dashed line is the best-fit King model found by Majewski et al. (2003) for the overall main body of the Sgr galaxy; the dotted line is the adopted best-fit model for Sgr,N and the continuous thick line is the sum of the last two models.

portion of the profile is reported in Tab. 1 1 and plotted in Fig. 2, together with the portions of the M54 and Sgr,N profiles derived by M05a.

The SB of Sgr,N is estimated at five radii. The three points at $R>10^{\prime \prime}$ nicely overlap with the observations by M05a, showing that the two profiles are in good agreement. The two points at $R<10^{\prime \prime}$ provide very strong new constraints on the fit of the overall profile. A $\mathrm{C}=1.90$ King model with $\mu_{V}(0)=15.4$ and $r_{c}=3.0^{\prime \prime}$ provides a good fit of the profile from the innermost observed point, at $R \simeq 3^{\prime \prime}$, to $R \sim 120^{\prime \prime}=2^{\prime}$; for $R>2^{\prime}$ the observed profile shows a much gentler decline with respect to the model, joining the flat profile of the core of Sgr at $R=r_{l} \simeq 10.5^{\prime}$ instead of $R=r_{t} \simeq 4.0^{\prime}$. While there is no particular physical reason in adopting a King model to represent Sgr,N, it provides an easy and satisfying way to parametrize its inner SB profile. Moreover, while the best-fit King model fails to reproduce the observed profile in the range $2^{\prime} \lesssim R \lesssim 10.5^{\prime}$, the fraction of the total Sgr,N light enclosed in this range amounts to just a few per cent; therefore the adopted model provides a reasonable description of the distribution of the bulk of the system light.

The derived parameters are listed in Tab. 2. Our results are fully compatible with the limits and the educated guesses by M05a; the most remarkable difference is in $r_{c}$ that was tentatively guessed as larger than that of M54 by M05a and, in fact, is found to be significantly smaller here. The absolute integrated $\mathrm{V}$ magnitude $\left(M_{V}=-7.8\right)$ is just slightly brighter than the upper limit by M05 $\left(M_{V} \leq-7.6\right)$. Assuming the best-fit models as correct, Sgr,N has a central V luminosity surface density that is slightly more than half that of M54, its total V luminosity is $\frac{1}{7}$ of the cluster and, finally both its core and half-light radii are $\sim$ one half of those of the cluster $^{4}$.

With these new and much tighter constraints on the light distribution of Sgr,N in hand, we will proceed in the following sections to perform a comparative study of its kinematics and those of M54.

\section{THE SPECTROSCOPIC SURVEY: OBSERVATIONS, DATA REDUCTION AND SELECTION OF THE SAMPLES}

The spectroscopic observations on which this study is based were obtained with the multi-object spectrographs DEIMOS on the Keck 2 telescope and FLAMES on the VLT-UT2 telescope, as listed in Tab. 3. The DEIMOS observations were undertaken using two different observing strategies: the first used the standard "slitlet" mode (the slitmask approach used by the DEEP2 team, e.g., Davis et al. 2003), where short slits of minimum length $4^{\prime \prime}$ and width $0^{\prime \prime} .7$ were milled; the second mode used smaller $1^{\prime \prime} .5$ long and $0^{\prime \prime} .7$ wide "holes" to allow larger multiplexing. In both cases, the high resolution 1200 lines/mm grating was employed with the OG550 blocking filter, giving a resolution of approximately $1.4 \AA$ FWHM. The slitlet spectra covered $\sim 6500$ $9000 \AA$ and were extracted and wavelength calibrated using the DEEP2 pipeline software; in contrast, the "holes" spectra were extracted using software developed by our own group (Ibata et al. 2005), with the final extracted spectra covering only the region $8400-8750 \AA$ (purely due to a limitation in the software).

For the DEIMOS observations we selected candidate M54/Sgr,N RGB and Red Clump (RC) stars lying within $R \lesssim 9^{\prime}$ from the center of the systems from the wide-field photometry of Monaco et al. (2002). A limiting magnitude of I = 18 was adopted, which ensures that we probe the Red Giant Branch to approximately one magnitude below the Red Clump (see Fig. 3).

Additional FLAMES observations were downloaded from the ESO archive; these targeted almost exclusively M54 stars, with only a small contamination from other populations. The FLAMES spectra were obtained with the high resolution setting HR21, which covers the calcium triplet from $8484-8757 \AA$, with a resolution of $0.5 \AA$ FWHM. The FLAMES data were extracted and calibrated using the "girBLDRS" pipeline ${ }^{5}$, developed for the European Southern Observatory by Geneva Observatory. Being of higher resolution, the FLAMES observations can be used to assess the accuracy of the DEIMOS data, but more importantly FLAMES is a fiber-fed spectrograph and is therefore immune to the velocity errors that can arise from mis-centering of slit spectrographs

\footnotetext{
${ }^{4} r_{h}$ is the half-mass radius of the best fitting King (1966) model: it can be considered equivalent to the half-light radius, in the present case. Note that using the same definition, the half-light radius of M54 is $r_{h}=0.79^{\prime}$, significantly larger than the value reported by Harris (1996, $\left.r_{h}=0.49^{\prime}\right)$. The latter number is very similar to what we obtain for the $r_{h}$ of the King (1962) empirical model that best-fits the profile of M54 (having the same C and $r_{c}$ adopted here), i.e. $r_{h}=0.47^{\prime}$. For C $\geq 1.0$ dynamical King (1966) models have $r_{h}$ larger than King (1962) empirical models by more than $10 \%$; the difference becomes larger than a factor of two for $\mathrm{C} \geq 2.0$. The half-light radius of the King (1962) best fitting the SB profile of Sgr, $\mathrm{N}$ is $r_{h}=0.23^{\prime}$. Hence independently of the adopted family of models, the half-light radius of M54 is a factor of $\sim 2$ larger than Sgr,N one. Preliminary tests suggest that available compilations of $r_{h}$ likely include quantities estimated in various non-homogeneous ways.

${ }^{5}$ See http://girbldrs. sourcef orge .net/
} 
TABLE 3

SPECTROSCOPIC OBSERVATIONS.

\begin{tabular}{cccc}
\hline Field & Instrument & Date & Exposure \\
\hline 1 & FLAMES Medusa 1 & $28 / 07 / 2005$ & $3 \times 845 \mathrm{~s}$ \\
2 & FLAMES Medusa 1 & $28 / 07 / 2005$ & $3 \times 845 \mathrm{~s}$ \\
3 & FLAMES Medusa 2 & $28 / 07 / 2005$ & $3 \times 845 \mathrm{~s}$ \\
4 & FLAMES Medusa 1 & $20 / 08 / 2005$ & $3 \times 795 \mathrm{~s}$ \\
5 & DEIMOS holes & $31 / 08 / 2005$ & $3 \times 900 \mathrm{~s}$ \\
6 & DEIMOS holes & $31 / 08 / 2005$ & $3 \times 900 \mathrm{~s}$ \\
7 & DEIMOS holes & $02 / 10 / 2005$ & $3 \times 950 \mathrm{~s}$ \\
8 & DEIMOS holes & $02 / 10 / 2005$ & $3 \times 950 \mathrm{~s}$ \\
9 & DEIMOS holes & $03 / 10 / 2005$ & $3 \times 720 \mathrm{~s}$ \\
10 & DEIMOS holes & $27 / 05 / 2006$ & $3 \times 300 \mathrm{~s}$ \\
11 & DEIMOS holes & $27 / 05 / 2006$ & $3 \times 300 \mathrm{~s}$ \\
12 & DEIMOS slitlets & $28 / 05 / 2006$ & $3 \times 720 \mathrm{~s}$ \\
13 & DEIMOS slitlets & $23 / 09 / 2006$ & $3 \times 300 \mathrm{~s}$ \\
14 & DEIMOS slitlets & $25 / 09 / 2006$ & $3 \times 300 \mathrm{~s}$ \\
\hline
\end{tabular}

such as DEIMOS.

The radial velocities of the target stars were obtained by cross correlating the observed spectra against an artificial template of the Ca II lines, using the same approach described and discussed in detail in Ibata et al. (2005) and Battaglia et al. (2008). The determination of the uncertainty on the velocity is based on a weighted sum of Gaussian fit errors to the individual $\mathrm{CaT}$ lines as described in detail in the thorough discussion by Battaglia et al. (2008), who also demonstrated that the velocity errors computed in this way are reliable. An additional sanity check was provided by the comparison with the scatter of the velocity measurements derived from the three individual Ca II lines separately (see, again, Battaglia et al. 2008).

The metallicity of the target stars were estimated from the combined equivalent width of the CaT lines. To this end we implemented the calibration described by Carrera et al. (2007), which it is claimed works well up to high $[\mathrm{Fe} / \mathrm{H}]$ values. As usual for CaT calibrations, $[\mathrm{Fe} / \mathrm{H}]$ is function of the combined equivalent width and of the difference between the $\mathrm{V}$ magnitude of the star and the mean level of the HB/RR Lyrae of the system $V-V_{H B}$. For full consistency with Carrera et al. (2007), the equivalent widths of the $\mathrm{CaT}$ lines were measured according to their prescriptions. The metallicity measurements were put on the Carretta \& Gratton (1997) scale, assuming $V_{H B}=\left\langle V_{R R L u}\right\rangle=18.17 \pm 0.01$ and $\mathrm{E}(\mathrm{B}-$ $\mathrm{V})=0.14$ (Layden \& Sarajedini 2000).

\subsection{Photometric properties of the sample}

From these data we constructed a final catalogue containing the radial velocity and the metallicity of 1152 stars in the innermost $15^{\prime} \times 10^{\prime}$ of the Sgr galaxy, as displayed in the lower panel of Fig. 3. The CMD of all these stars is shown in the upper panel of the same figure. The RGB sequences of the two systems are quite well separated in the CMD down to the faintest stars included in our sample $(I \simeq 18)$. However, we also selected stars lying on the Red Clump of the Sgr,N population, at $V-I \simeq 1.15$ and $16.8<I<17.3$ : this feature overlaps with the RGB of M54, hence we will need some further information to establish the membership of these stars, in addition to their color, magnitude and radial velocity. In particular, the metallicity would be very useful to disentangle the two populations in this range. M54 has $[\mathrm{Fe} / \mathrm{H}]=-1.55$ (Brown. Wallerstein \& Gonzalez 1999; Da Costa \& Armandroff 1995), with a small dispersion (see Sarajedini \& Layden 1995, and Sect. 3.4, below). In contrast, the metallicity distribution of the Sgr galaxy is dominated by a wide peak at $[\mathrm{Fe} / \mathrm{H}] \simeq$ -0.4 (see Monaco et al. 2005b; Bonifacio et al. 2006; Bellazzini et al. 2006a, and references therein), extending from $[\mathrm{Fe} / \mathrm{H}] \sim-1.0$ to $[\mathrm{Fe} / \mathrm{H}] \sim 0.0$. Stars more metal poor than $[\mathrm{Fe} / \mathrm{H}] \sim-1.2$ are quite rare and, presumably, they would pass their core-Helium-burning phase as RR Lyrae or Blue Horizontal Branch stars (Monaco et al. 2003): hence Red Clump stars of Sgr should have $[\mathrm{Fe} / \mathrm{H}] \gtrsim-1.0$, much more metal-rich than any M54 star.

As a first step for the selection of two samples representative of the Sgr,N and M54 populations, we introduce the photometric classification defined by the three curves overplotted on the CMD of Fig. 3. Proceeding from left (blue) to right (red) we assigned the flag $\mathrm{cmd}$ in the following way: $c m d=1$ to the stars enclosed between the first and second curve, as likely M54 members, $\mathrm{cmd}=2$ to the stars enclosed between the second and third curve, as likely Sgr,N members, and $c m d=0$ to stars lying to the blue of the first curve or to the red of the third curve. A check a-posteriori has shown that all the stars having $c m d=0$ have radial velocities incompatible with being members of Sgr,N or M54, thus supporting the validity of our photometric classification.

As the coordinate of the centers of M54 and Sgr,N we adopt $\alpha_{0}=283.763750^{\circ}$ and $\delta_{0}=-30.478333^{\circ}$ from Noyola \& Gebhardt (2006), and we convert to cartesian coordinates $\mathrm{X}, \mathrm{Y}$ (in arcmin) projecting the equatorial coordinates of each star $(\alpha, \delta)$ on the plane of sky as in van de Ven et al. (2006)

$$
\begin{gathered}
X=-(10800 / \pi) \cos (\delta) \sin \left(\alpha-\alpha_{0}\right) \\
Y=(10800 / \pi)\left[\sin (\delta) \cos \left(\delta_{0}\right)-\cos (\delta) \sin \left(\delta_{0}\right) \cos \left(\alpha-\alpha_{0}\right)\right]
\end{gathered}
$$

with $\mathrm{X}$ increasing toward the West and Y increasing toward the North. Adopting the distance to Sgr and M54 measured by Monaco et al. (2004), 1 arcmin corresponds to $7.65 \mathrm{pc}$.

\subsection{Comparison between independent measures}

As there are several stars in common between the observational sets taken with different instruments and or set-ups, we have the opportunity to check the consistency and the accuracy of our $V_{r}$ and $[\mathrm{Fe} / \mathrm{H}]$ estimates. In Fig. 4 we show the comparison between $V_{r}$ estimates from the various sources: $V_{f}^{F}$ are the velocities obtained with FLAMES/fibers, $V_{h}^{D}$ are from DEIMOS/holes, and $V_{s}^{D}$ are from DEIMOS/slits. It can be readily appreciated that the consistency among the different sets of measures is excellent (i.e., $\Delta V_{r} \simeq 0.0 \mathrm{~km} / \mathrm{s}$ ). The typical accuracy, as measured from the r.m.s. of the $V_{r}$ differences is $\leq \pm 2.0 \mathrm{~km} / \mathrm{s}$, that is satisfying for the present purpose. The actual uncertainty on the single measure should be a factor $\sqrt{2}$ smaller than the r.m.s. of $\Delta V_{r}$ (i.e. $\simeq \pm 1.4 \mathrm{~km} / \mathrm{s}$ ), as the latter includes the uncertainties of both the estimates, added in quadrature. Of the 414 velocity differences plotted in Fig. 4 just $\sim 10$ are significantly larger than $\pm 3.0 \mathrm{~km} / \mathrm{s}$ : some of these may be associated with binary system observed at different orbital phases (see Monaco et al. 2007). 


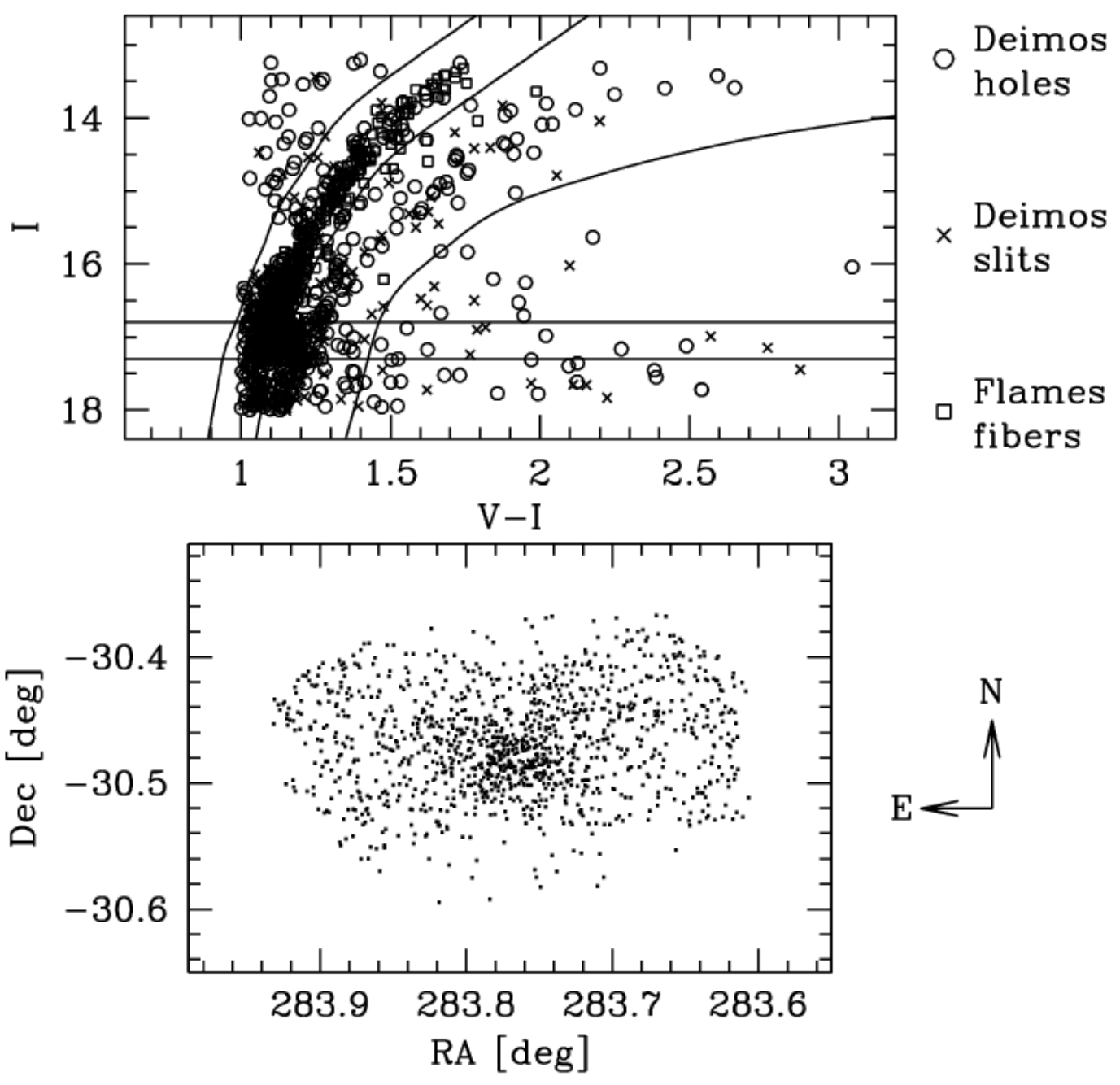

FIG. 3.- CMD (upper panel) and distribution in the sky (lower panel) of the 1152 stars constituting our final sample. Different colors/symbols are associated to measures obtained with different instruments and/or set-ups. The curves in the CMD show the photometric selection box adopted for a first discrimination between candidate M54 or Sgr,N members.

Fig. 5 shows the comparison between $[\mathrm{Fe} / \mathrm{H}]$ estimates. In this case, while the consistency between measures from FLAMES and DEIMOS/holes spectra is very good, $[\mathrm{Fe} / \mathrm{H}]$ from DEIMOS/slits spectra are $\sim 0.08$ dex larger - in average - than those from the other two sources. We were unable to find the cause of this mismatch and to preserve the homogeneity of the final merged dataset we corrected all the $[\mathrm{Fe} / \mathrm{H}]$ values from DEIMOS/slits spectra by adding -0.08 to all of them. The overall accuracy is more than satisfying, with a typical r.m.s of 0.1 dex, and also in this case the outliers are rare.

Finally Fig. 6] shows the comparison between our final $V_{r}$ and $[\mathrm{Fe} / \mathrm{H}]$ estimates and those obtained by Monaco et al. (2005b) from high-resolution FLAMESUVES@VLT spectroscopy, for the seven stars in common between these datasets. The agreement in the radial velocity is good: if the only outlier (at $\Delta V_{r} \sim-10 \mathrm{~km} / \mathrm{s}$ ) is excluded the r.m.s. of the difference is just $2.3 \mathrm{~km} / \mathrm{s}$ and the average difference is zero ${ }^{6}$. Therefore the reliability and the accuracy of our radial velocity scale is fully confirmed. The comparison of metallicities is more difficult to interpret and would have benefited by a larger number of stars in common between the two samples. For four of the seven stars, ranging from $[\mathrm{Fe} / \mathrm{H}] \simeq-1.5$ to $[\mathrm{Fe} / \mathrm{H}] \simeq-0.2$ the agreement is more than acceptable, given the associated uncertainties. The other three stars show a considerable $\Delta[\mathrm{Fe} / \mathrm{H}]$, but one of them was reported to have a tentative $[\mathrm{Fe} / \mathrm{H}]$ estimate from Monaco et al. (2005b) because its spectrum was affected by molecular bands making the analysis less reliable. In any case, none of the observed differences are so large as to cause us to erroneously classify a Sgr,N star as a M54

6 Also in this case we suggest multiplicity as the most likely origin of the outlier (see, again, Monaco et al. [2007). Note that binaries are not expected to significantly affect the observed velocity dispersions in systems like those considered here (see Hargreaves et al. 1996; Olszewski et al. 1996, for detailed discussion). 


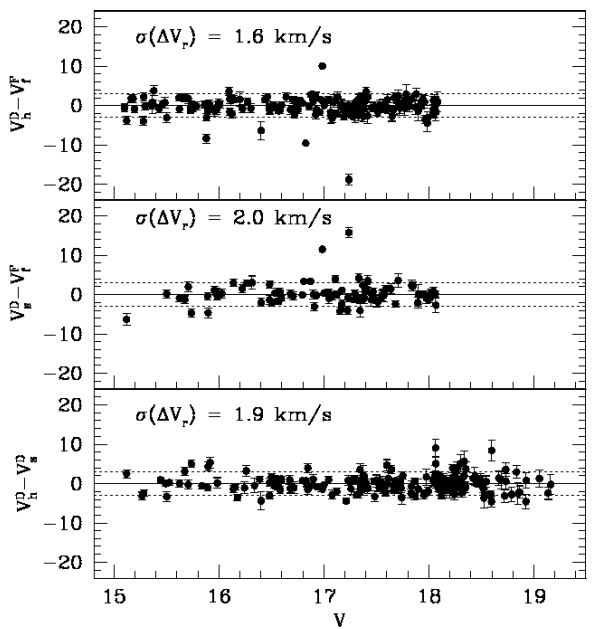

FIG. 4.- Comparison between independent $V_{r}$ estimates obtained from spectra acquired with different telescopes/instruments/set-ups. Each panel reports the differences between the $V_{r}$ estimates as a function of $\mathrm{V}$ magnitude. The $\pm 3.0 \mathrm{~km} / \mathrm{s}$ range around zero is enclosed by the two dotted lines. Upper panel: DEIMOS/holes vs. FLAMES/fibers (160 stars); middle panel: DEIMOS/Slits vs. FLAMES/fibers (80 stars); lower panel: DEIMOS/holes vs. DEIMOS/Slits. The standard deviation of each set of $\Delta V_{r}$, computed after recursive clipping of the very few 2.5- $\sigma$ outliers, is reported in each panel.

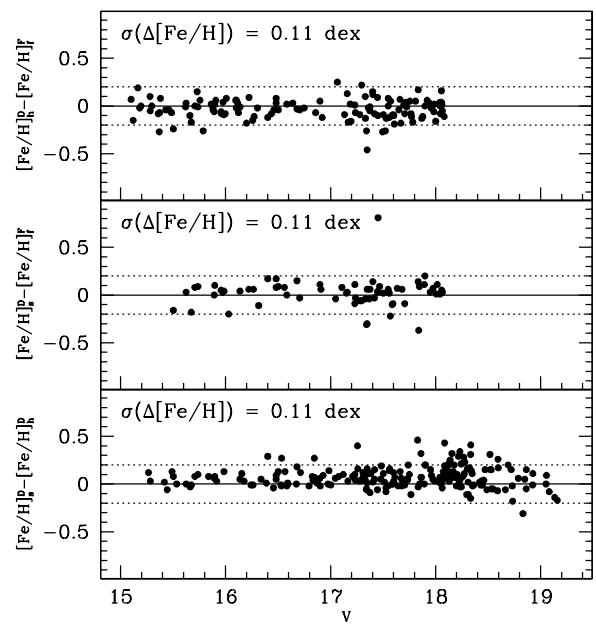

FIG. 5.- Comparison between independent $[\mathrm{Fe} / \mathrm{H}]$ estimates obtained from spectra acquired with different telescopes/instruments/set-ups. Each panel reports the differences between the $[\mathrm{Fe} / \mathrm{H}]$ estimates as a function of $\mathrm{V}$ magnitude. The \pm 0.2 dex range around zero is enclosed by the two dotted lines. Upper panel: DEIMOS/holes vs. FLAMES/fibers; middle panel: DEIMOS/Slits vs. FLAMES/fibers; lower panel: DEIMOS/holes vs. DEIMOS/Slits. The standard deviation of each set of $\Delta[\mathrm{Fe} / \mathrm{H}]$ is reported in each panel.

member, or vice-versa, according to the selection criteria that are adopted below. Taking also into account the high degree of self-consistency among different sets of measures shown in Fig. 5, we conclude that also our metallicity scale is sufficiently reliable and accurate for the purposes of the present analysis.
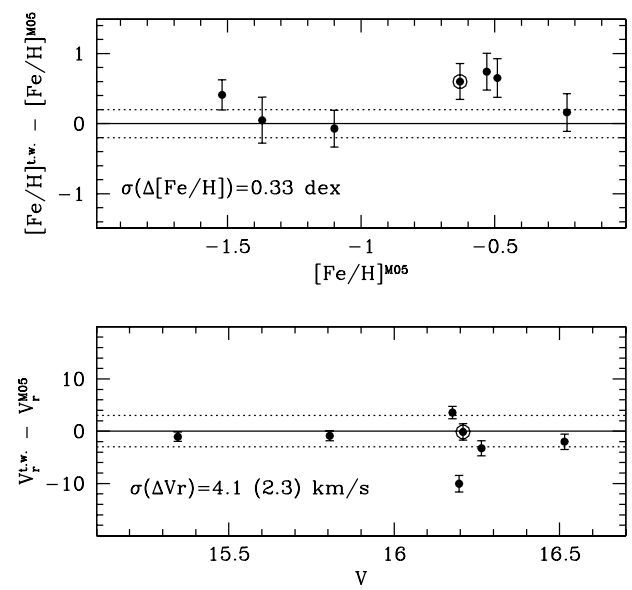

FIG. 6.- Comparison between the $[\mathrm{Fe} / \mathrm{H}]$ and $V_{r}$ estimates obtained in this work (t.w.) and in Monaco et al. (2005b, M05), for the seven stars in common. The encircled dot correspond to a star with molecular bands in its UVES spectrum, whose metallicity is classified as tentative by Monaco et al. (2005b). In the lower panel, the standard deviation value reported in parentheses refers to the sub-sample from which the outlier at $\Delta V_{r} \simeq 10 \mathrm{~km} / \mathrm{s}$ has been removed. The dotted lines encloses the range $0.0 \pm 0.2$ dex in the upper panel, and $0.0 \pm 3.0 \mathrm{~km} / \mathrm{s}$ in the lower panel.

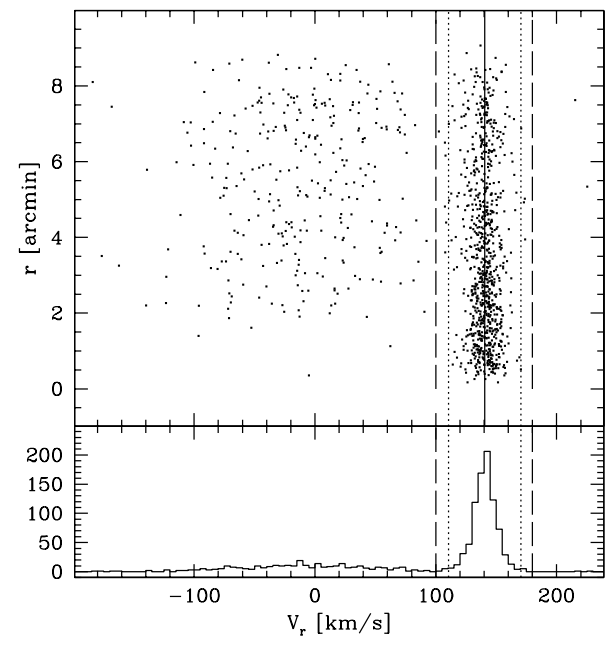

FIG. 7.- Radial velocity of program stars as a function of distance from the center of Sgr,N/M54 (upper panel), and radial velocity distribution (lower panel). The long dashed lines mark the range we adopted for the first selection of candidate Sgr/M54 members. The dotted lines enclose the (global) $\pm 3 \sigma$ range from the mean of the selected samples of candidates (continuous line).

\subsection{Radial velocity distribution}

The distribution of the radial velocity of all the observed stars as a function of their (projected) distance $\left(r=\sqrt{X^{2}+Y^{2}}\right)$ from the center of the system is shown in Fig. 7. Two very different populations can be identified in this plot: a broadly-distributed cloud of Galactic field stars showing a large dispersion around $V_{r} \sim 0$ $\mathrm{km} / \mathrm{s}$, and an abundant low-dispersion population with a systemic $V_{r} \sim 141 \mathrm{~km} / \mathrm{s}$, typical of M54 and Sgr,N. As a first broad selection, and following Ibata et al. (1997), 


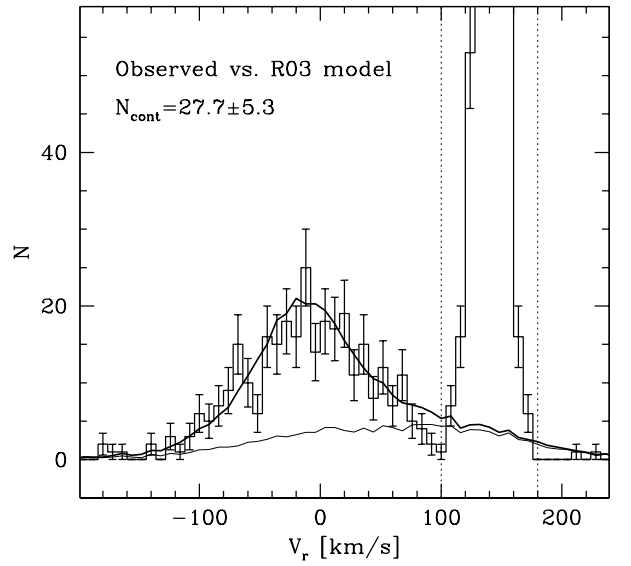

FIG. 8.- Zoomed view of the observed radial velocity distribution (histogram with noise error bars) around the peak of the Galactic field population. The thick curve is the distribution of all Galactic field stars with color and magnitude similar to program stars predicted by the R03 Galactic model in the considered direction, shifted by $-8 \mathrm{~km} / \mathrm{s}$ to match the position of the observed peak and rescaled to minimize the $\chi^{2}$ value with respect to the observations for $V_{r}<100.0 \mathrm{~km} / \mathrm{s}$. The thin curve shows the distribution of (only) giant stars from the model, with the same normalization. The vertical dotted lines enclose the $100 \mathrm{~km} / \mathrm{s}<V_{r}<180$ $\mathrm{km} / \mathrm{s}$ interval. The expected number of Galactic stars falling in this velocity range $N_{\text {cont }}$ is reported.

we retained as possible M54/Sgr,N members all the (832) stars having $100 \mathrm{~km} / \mathrm{s} \leq V_{r} \leq 180 \mathrm{~km} / \mathrm{s}$ (dashed lines in Fig. 7). We then computed the mean and the dispersion of this sample: the $\pm 3 \sigma$ range around the global mean is enclosed within the two dotted lines. We do not reject the stars outside the $3 \sigma$ range at this stage, since the velocity distribution is different at different radii (see below), and so a more refined choice is to exclude $>3 \sigma$ outliers in any considered radial bin. However, in Sect. 4 we will see that the adopted bin-by-bin $3 \sigma$ rejection criterium will exclude all of these stars.

Fig. 8 shows that we expect some (limited) contamination from Milky Way stars even in our $V_{r}$ selected sample. The thick continuous line superposed on the observed histogram of the radial velocities zoomed on the Galactic component, shows the excellent agreement between the observations and the predictions of the Robin et al. (2003) Galactic model, once it has been shifted by -8.0 $\mathrm{km} / \mathrm{s}$ to match the observed peak and rescaled to minimize $\chi^{2}$ for $V_{r}<100.0 \mathrm{~km} / \mathrm{s}$. The model suggests that up to $\sim 30$ Milky Way stars may be present even in the relatively narrow $V_{r}$ window we have adopted to select M54/Sgr,N stars. It is interesting to note that among the stars having $100 \mathrm{~km} / \mathrm{s}<V_{r}<180 \mathrm{~km} / \mathrm{s}$ but lying outside of the $\left\langle V_{r}\right\rangle \pm 3 \sigma$ range, seven have $V_{r}<\left\langle V_{r}\right\rangle-3 \sigma$ and four have $\left.V_{r}\right\rangle\left\langle V_{r}\right\rangle+3 \sigma$, in agreement with the trend with $V_{r}$ predicted by the model. Fig. 8 shows also that most of the Galactic interlopers are expected to be giants, according the the Robin et al. (2003) Galactic model. However, it is likely that such stars should be - in general much closer to us than Sgr: the model finds that the contaminating giants have a mean distance of $8.7 \mathrm{kpc}$ (corresponding to $\Delta(M-m)=+2.4 \mathrm{mag}$, with respect to Sgr), $90 \%$ of them have $D \leq 11 \mathrm{kpc}$ and $99 \%$ of them have
$D \leq 14 \mathrm{kpc}$, i.e. much lower than $D_{S g r}=26.3 \mathrm{kpc}$. For this reason the difference between their $\mathrm{V}$ magnitude and $V_{H B}$ of Sgr must be a bad proxy for their gravity. The typical spectroscopic metallicity error incurred by overestimating the distance modulus of these Galactic stars by $2.4 \mathrm{mag}$ is $\Delta[\mathrm{Fe} / \mathrm{H}]=-0.58$, so the stars will appear to be less metal-rich than they are in reality while their color and magnitude mimic those of genuine Sgr/M54 stars. As a consequence, for a Galactic star the metallicity obtained from the Calcium Triplet $(\mathrm{CaT})$ technique would normally be much different from what would be expected from the magnitude and color measurements. Therefore, there is some hope to exclude these Galactic interlopers from our final samples, identifying them by their "odd" color-metallicity combination, as we will do in our final selection (Sect. 3.5, below). As the mean metallicity of contaminating giants is $\langle[\mathrm{Fe} / \mathrm{H}]\rangle=-0.75$ and $90 \%$ of them have $[\mathrm{Fe} / \mathrm{H}]<-0.33$ it is quite likely that many of them would have a measured metallicity lower than the lower threshold we will assume for Sgr,N members $([\mathrm{Fe} / \mathrm{H}] \geq-0.8)$. On the other hand those spuriously showing metallicities compatible with M54 will probably be too red to be selected as possible cluster members (Sect. 3.5 and Fig. 3i).

Finally it is worth noting that the spectra of all the 843 stars that passed the selection in $V_{r}$ have Signal to Noise ratio (per pixel) $S / N>12 ; 300$ of them have $S / N>50$ and 63 of them have $S / N>100$ (see Fig. 9d).

\subsection{Metallicity distribution}

The metallicity distribution of the velocity selected stars is shown if Fig. 9a. The presence of two populations is very clear: there is a metal poor peak around $[\mathrm{Fe} / \mathrm{H}] \simeq-1.5$ that must be dominated by M54 stars (Brown, Wallerstein \& Gonzalez 1999) and a broader distribution extending from $[\mathrm{Fe} / \mathrm{H}] \gtrsim-1.0$ to supersolar metallicity corresponding to the main population of Sgr,N (see Monaco et al. 2005b; Bellazzini et al. 2006a; Bonifacio et al. 2006, and references therein). As mentioned earlier, in the present context we will use the metallicity just as a further means to select samples of M54 and Sgr,N members that are as clean as possible, so as to allow further analysis of their kinematic properties. Therefore, we limit the discussion of the metallicity to the brief considerations listed below.

1. We confirm that the bulk of Sgr stars have $[\mathrm{Fe} / \mathrm{H}]>-1.0$, belonging to a broad distribution peaking around $[\mathrm{Fe} / \mathrm{H}]=-0.4$ and reaching super-solar metallicities (Monaco et al. 2005b; Bonifacio et al. 2006). Monaco et al. (2003) estimated that in the main body of the Sgr galaxy old metal poor stars $([\mathrm{Fe} / \mathrm{H}] \lesssim-1.2)$ should provide less than $12 \%$ of the whole stellar content. Several arguments suggest that this fraction should be even lower in the very central region considered here (see Alard 2001; Bellazzini et al. 2006b; Siegel et al. 2007, and references therein). However, we note that there are four stars having $[\mathrm{Fe} / \mathrm{H}]<-2.0$ in our sample (see Fig. 9) that are probably too metal poor to be members of M54 and might be part of the metal-poor population of Sgr. 


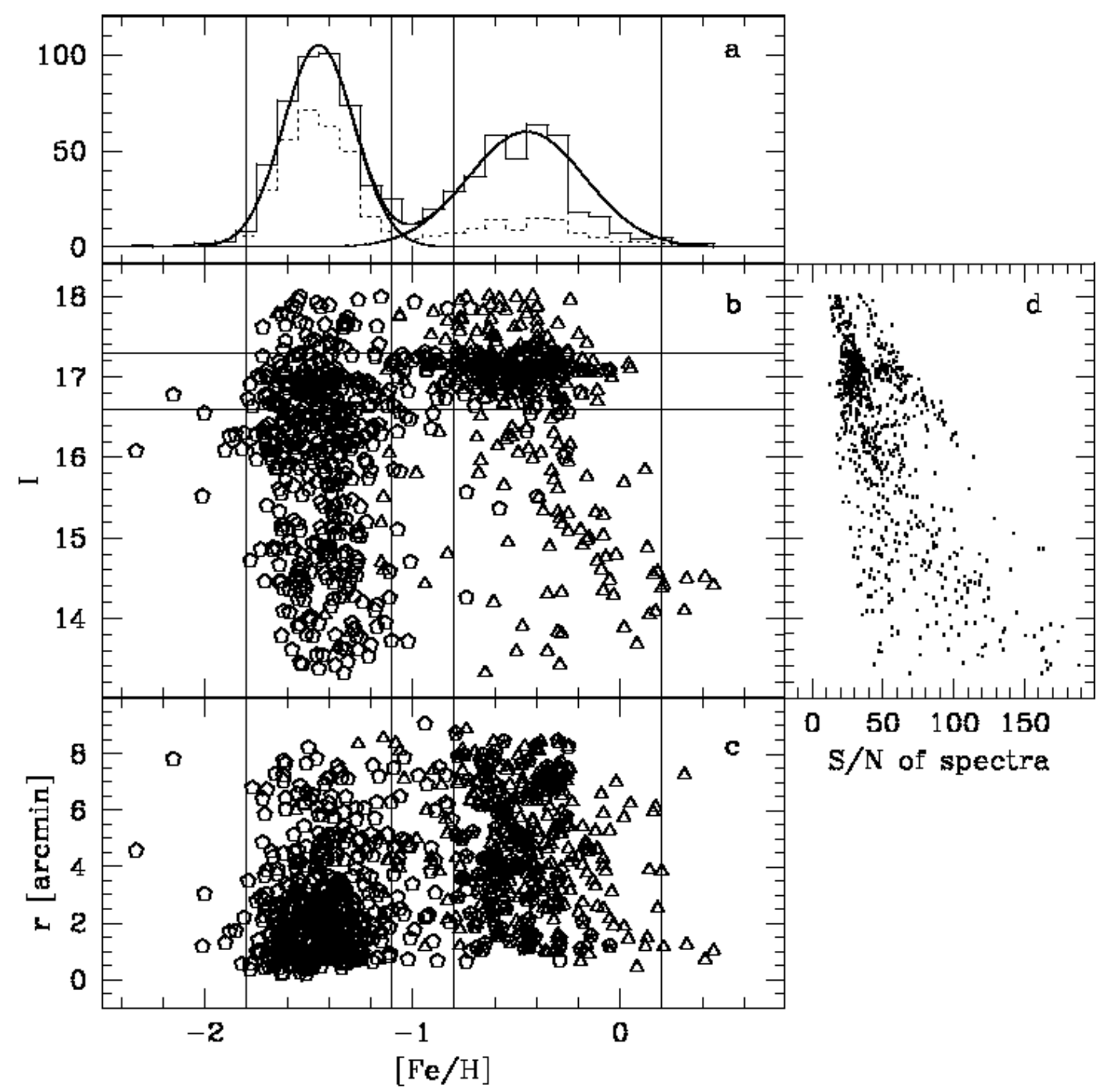

FIG. 9.- Selection of clean samples of M54 and Sgr,N members. Panel a: metallicity distribution of the 843 stars that survived the selection in radial velocity. The superposed curve is the sum of two Gaussian distributions having mean and standard deviation $(\langle[\mathrm{Fe} / \mathrm{H}]\rangle, \sigma)=(-0.45,0.28)$ and $(-1.45,0.17)$, representing the main populations of Sgr, N and M54, respectively. The dotted histogram is the observed distribution for stars having $R \leq 3.0^{\prime}$. Panel $b$ : metallicity vs. I magnitude; open triangles are stars having $c m d=2$, i.e. photometrically selected as likely Sgr members (see Fig. 3); open pentagons are stars with $\mathrm{cmd}=1$, i.e. photometrically selected as likely M54 members. The horizontal lines enclose the Sgr RC magnitude range $(16.6<I<17.3)$. The vertical lines mark the adopted thresholds in metallicity: $-1.8 \leq[\mathrm{Fe} / \mathrm{H}] \leq-1.1$, for the M54 sample; $-0.8 \leq F e / H] \leq+0.2$ for the Sgr,N sample. Panel c: metallicity vs. distance from the center of M54. The symbols are the same as in the above panel, except for asterisks superposed on open pentagons: these are stars that lie on the RGB of M54 in the CMD but in fact belong to the superposed RC of Sgr, i.e. they have $16.6<I<17.3$ and a metallicity typical of Sgr members. Panel d: Signal to Noise (per pixel) of the spectra as a function of I magnitude. All the spectra have $S / N>12$.

2. The peak of the metallicity distribution corresponding to M54 is best-fitted by a Gaussian curve having mean $\langle[\mathrm{Fe} / \mathrm{H}]\rangle=-1.45$. This is slightly higher $(\sim+0.1)$ than what was found by Brown. Wallerstein \& Gonzalez (1999) and by Da Costa \& Armandroff (1995), while it is in good agreement with Armandroff (1989). Note that any possible small shift in the zero point of our metallicity scale does not affect the accuracy of the metallicity ranking, that is $\simeq \pm 0.1$ and it is the relevant figure in the present context.

3. Based on the analysis of the Color - Magnitude distribution of RGB stars, Sarajedini \& Layden
(1995) concluded that there is an intrinsic metallicity spread among M54 stars of $\sigma_{i n t}([\mathrm{Fe} / \mathrm{H}])=$ 0.16 dex. Using spectroscopic metallicities obtained with the CaT technique for five M54 members, Da Costa \& Armandroff (1995) found further support for this hypothesis (but see Brown, Wallerstein \& Gonzalez 1999). Our much larger sample of CaT metallicities provides new supporting evidence for this possibility. The sample with $-1.0 \leq[\mathrm{Fe} / \mathrm{H}] \leq-2.0$ has an observed standard deviation of 0.17 dex, larger than what is expected from the statistical scatter (see Fig. 5, above). Deconvolving the (internal) r.m.s. 



Fig. 10.- Upper left panel: CMD of stars selected to belong to the final Sgr,N (open triangles) and M54 (open pentagons) samples, superposed on the CMD of all stars having $R<9^{\prime}$ from Monaco et al. (2002, small dots). The thin horizontal lines enclose the Red Clump of Sgr $(16.6<I<17.3)$. The position in the sky of the selected stars relative to the center of Sgr,N/M54 is plotted in the lower left panel, the symbols are the same as above. The radial distributions of stars selected on the Sgr, $\mathrm{N}$ sequences (continuous histogram) and on the M54 sequences (dotted line) are plotted in the lower right panel The upper right panel shows the uncertainties of the $V_{r}$ estimates as a function of I magnitudes for the selected stars.

scatter (0.11 dex, see Fig. 5) from the observed width of the distribution we obtain an estimate of the intrinsic spread of $\sigma_{\text {int }}([\mathrm{Fe} / \mathrm{H}])=0.14 \mathrm{dex}$, in good agreement with previous estimates. It seems very unlikely that the width of the M54 peak is significantly contaminated by Sgr stars, as the width remains unchanged if we limit the sample to the innermost $3^{\prime}$ where M54 should dominate the population mix. If finally confirmed by high resolution spectroscopy of a large sample of stars, the purported metallicity spread would constitute another element of similarity between M54 and other large-size very bright clusters like $\omega$ Cen, G1 and the like, that are also suspected of being remnant nuclei of disrupted galaxies (see Mackev \& van den Bergh 2005; Federici et al. 2007; Catelan 2007; Maschenko \& Sills 2005; Böker

\section{7, and references therein).}

We think that the presently available metallicity estimates provide a metallicity ranking that is more than sufficient for the purposes of the present paper, i.e. (a) to discriminate M54 stars from Sgr,N stars in the magnitude range where the two population overlaps in the CMD $(16.8<I<17.3)$, and (b) to exclude possible interlopers as stars having the "wrong" metallicity for their color. The details of the adopted selections are described in the following sub-section.

\subsection{The final selections}

The scheme of our finally adopted selections is illustrated in Fig. 9b,c. Our aim is to have a sample of M54 stars and a sample of Sgr,N stars as clean as possible from any kind of interlopers. The adopted criteria are the following: 
- We accept as members of the M54 sample the stars having $\mathrm{cmd}=1$ and $-1.8 \leq[\mathrm{Fe} / \mathrm{H}]<-1.1$.

- We accept as members of the Sgr,N sample the stars having $\mathrm{cmd}=2$ and $-0.8 \leq[\mathrm{Fe} / \mathrm{H}] \leq+0.2$, or having $\mathrm{cmd}=1$ and $-0.8 \leq[\mathrm{Fe} / \mathrm{H}] \leq+0.2$ if $16.6<I<17.3$ (Sgr RC stars superposed on the RGB of M54).

The metallicity separation avoids mixing between samples. The requirement that the $\mathrm{cmd}$ flag and the metallicity broadly agree in assigning the membership is likely to exclude most of the Galactic interlopers. In the region in which the RC of Sgr,N and the RGB of M54 overlap, the cmd flag does not provide any discrimination and we rely just on the metallicity. We have adopted quite conservative criteria to obtain very reliable samples of genuine Sgr,N and M54 stars. As a further check, all the results presented in the following about the kinematics of $\mathrm{Sgr}, \mathrm{N}$ have been verified to hold also when subsamples in which stars in the RC region were excluded are adopted. The main properties of the selected samples are shown in Fig. 10.

With the selection described above we select a sample of 425 very likely M54 members and of 321 very likely $\mathrm{Sgr}, \mathrm{N}$ members. In the process of clipping $3 \sigma$ velocity outliers in any considered radial bin (which will be performed in Sect. 4, below), we will further exclude eight stars from the M54 sample and three stars from the Sgr,N sample. Hence the actual detailed analysis of the kinematics of the two systems will be performed on 417 stars for M54 and on 318 stars for Sgr,N.

\section{THE KINEMATICS OF M54 AND SGR,N: OBSERVATIONAL FACTS}

In Tab. 4 we list the estimates of some relevant physical parameters of M54 that are available in the literature. For the structural parameters $\left(r_{c}, r_{t}\right.$, etc.) we will preferentially adopt those from Trager. King \& Diorgovski (1995), since the best-fit model proposed by these authors is in good agreement with the profile obtained from star counts in the outer regions of the cluster by M05a. However in some cases we will consider the effects of the adoption of different sets of parameters on our results. It is reassuring that most authors derived quite similar parameters from very different datasets. All the available mass estimates for M54, also obtained with different methods and from different datasets, range between $1.0 \times 10^{6} M_{\odot}$ and $2.0 \times 10^{6} M_{\odot}$ (Illingworth 1976; Mandushev. Staneva \& Spasova 1991; Pryor \& Meylan 1993; Mackey \& Gilmore 2003; McLaughlin \& van der Marel 2005).

In Tab. 5 we report the average kinematical properties of the M54 and Sgr,N samples as a whole. The errors on all the reported quantities have been obtained by a jackknife bootstrapping technique (Lupton 1993). The plain average $V_{r}$ of the two samples differs by $1.5 \pm 0.7$ $\mathrm{km} / \mathrm{s}$, that is, just a $\sim 2 \sigma$ difference. However an average with iterative $2-\sigma$ clipping seems a much more appropriate estimator of the systemic velocity since it is very robust to outliers and is more effective in estimating the mode of the samples. The sigma clipping process converges very fast in both cases, leaving very rich final samples $\left(N_{2 \sigma}=246\right.$ and 348, for Sgr,N and M54,

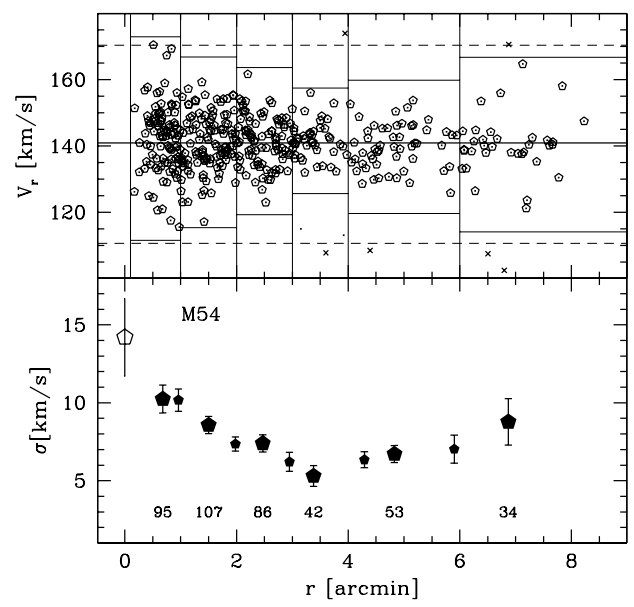

FIG. 11. - Velocity dispersion profile of M54 stars. The upper panel shows the $V_{r}$ distribution as a function of distance from the cluster center for individual stars of the M54 sample. Only stars plotted as dots encircled by open pentagons are retained for the computation of $\sigma$ in the various radial bins: small dots alone are stars rejected only because they are "local" $3 \sigma$ outliers of the bins, crosses are stars that would have been rejected also as $3 \sigma$ outliers of the whole $\mathrm{Sgr}, \mathrm{N}+\mathrm{M} 54$ sample (the global $\pm 3 \sigma$ range is enclosed by the long-dashed lines). The vertical lines display the adopted independent bins, of variable size. The global mean is marked by the continuous horizontal thick line. The lower panel displays the actual Velocity dispersion profile. The large filled pentagons are the dispersions estimated in the corresponding bins displayed in the upper panel, with their bootstrapped errors. The number of stars per bin is also reported below the points. The small filled pentagons are the estimates in the additional, partially overlapping, bins. The open pentagon is the estimate of $\sigma$ at the center of M54 obtained by Illingworth (1976) from integrated spectroscopy.

respectively). The difference in the clipped mean velocities is a mere $0.8 \mathrm{~km} / \mathrm{s}$. We conclude that the Sgr,N and M54 do coincide also in line of sight (l.o.s.) velocity, a further proof in support of their spatial coincidence. Many authors have noted before that the systemic velocity of Sgr and M54 are very similar (Ibata et al. 1997; Da Costa \& Armandroff 1995; (Monaco et al.|2005b); we think we have conclusively established here that M54 and the nucleus of Sgr have the same systemic l.o.s velocity, within $\simeq \pm 1.0 \mathrm{~km} / \mathrm{s}$.

The velocity dispersions of the whole Sgr,N and M54 do not seem very different, at a first glance. However, it should be recalled here that in the radial range covered by our data $\left(r \leq 9^{\prime}\right)$, the surface brightness of M54 and $\mathrm{Sgr}, \mathrm{N}$ declines by more than $\sim 8 \mathrm{mag} / \mathrm{arcsec}^{2}$. Hence, in the present context, what is really relevant is the comparison between velocity dispersion profiles as a function of distance from the center of the system, which will be presented in the following section.

\subsection{Velocity dispersion profiles}

In the upper panel of Fig. 11 we show the distribution of the M54 stars in the $V_{r}$ vs. $r$ plane. We have divided the sample into two sets of six and five independent radial bins of different size, respectively, in order to keep the number of stars per bin as high as possible while maintaining the highest degree of spatial resolution (the primary set of independent bins corresponds to the odd rows of Tab. 6, the secondary set corresponds to the even 
TABLE 4

LitERATURE DATA FOR M54.

\begin{tabular}{|c|c|c|c|c|c|c|c|}
\hline $\begin{array}{c}r_{c} \\
\operatorname{arcmin}\end{array}$ & $\begin{array}{c}r_{t} \\
\operatorname{arcmin}\end{array}$ & $\bar{C}$ & $\begin{array}{c}\mu_{V}(0) \\
\mathrm{mag} / \operatorname{arcsec}^{2}\end{array}$ & $\begin{array}{c}V_{t} \\
\mathrm{mag}\end{array}$ & $\begin{array}{c}{[\mathrm{Fe} / \mathrm{H}]} \\
\operatorname{dex}\end{array}$ & method & Ref. \\
\hline 0.11 & 7.4 & 1.83 & 14.90 & $\begin{array}{l}7.61 \\
7.68\end{array}$ & & $\begin{array}{l}\text { g.b. ap. phot. } \\
\text { g.b. ap. phot. }\end{array}$ & $\begin{array}{c}\frac{\text { Illingworth \& Illingworth }}{\text { Peterson (1993) }} \\
\text { (1976) }\end{array}$ \\
\hline$\lesssim 0.11$ & 7.4 & 1.84 & 14.75 & & $\begin{array}{l}-1.55 \\
-1.55\end{array}$ & $\begin{array}{l}\text { g.b. ap. phot. } \\
\text { CaT spectr. } \\
\text { HR spectr. } \\
\text { HST phot. }\end{array}$ & $\begin{array}{c}\text { Trager, King \& Diorgovski }(1995) \\
\text { Da Costa \& Armandroff }(1995) \\
\text { Brown, Wallerstein \& Gonzalez (1999) } \\
\text { Mackey \& Gilmore (2003) }\end{array}$ \\
\hline $\begin{array}{l}\widetilde{0} 09 \\
0.05\end{array}$ & 9.9 & 2.04 & $\begin{array}{l}14.35 \\
14.12\end{array}$ & & & $\begin{array}{l}\text { g.b. ap. phot. } \\
\text { HST phot. }\end{array}$ & $\begin{array}{c}\text { McLaughlin \& van der Marel (2005) } \\
\text { Novola \& Gebhardt (2006) }\end{array}$ \\
\hline
\end{tabular}

Note. - g.b. ap. phot. = ground based aperture photometry; HST phot. = integrated photometry and/or star counts from Hubble Space Telescope data; CaT spectr. = medium resolution spectroscopy using the infrared Calcium Triplet as metallicity indicator; HR spectr. = High Resolution spectroscopy and elemental abundance analysis. See also Webbink (1985), Harris (1996) and Mackey \& Gilmore (2003) for collections of literature data on M54.

TABLE 5

Average Kinematic properties of the M54 and SGR,N SAMPLES.

\begin{tabular}{lcccccc}
\hline & $\mathrm{N}_{\text {tot }}$ & $\begin{array}{c}\left\langle V_{r}\right\rangle \\
\mathrm{km} / \mathrm{s}\end{array}$ & $\begin{array}{c}\sigma \\
\mathrm{km} / \mathrm{s}\end{array}$ & $\begin{array}{c}\sigma^{*} \\
\mathrm{~km} / \mathrm{s}\end{array}$ & $\begin{array}{c}\mathrm{N}_{2 \sigma} \\
\left\langle V_{r}\right\rangle_{2 \sigma} \\
\mathrm{km} / \mathrm{s}\end{array}$ \\
\hline Sgr,N & 321 & $139.4 \pm 0.6$ & $10.0 \pm 0.5$ & $9.6 \pm 0.4$ & 246 & 139.9 \\
$\mathrm{M} 54$ & 425 & $140.9 \pm 0.4$ & $9.3 \pm 0.5$ & $8.3 \pm 0.3$ & 348 & 140.7 \\
\hline
\end{tabular}

NoTE. - $\mathrm{N}_{2 \sigma}=$ number of stars in the sample once the iterative $2 \sigma$ clipping algorithm to compute $\left\langle V_{r}\right\rangle_{2 \sigma}$ has converged, i.e. there is no more $2 \sigma$ outlier. Note that at each iteration the algorithm adopts the current value of $2 \sigma$ that is, in general, significantly lower than the global value reported in the table. $\sigma^{*}$ are the global values of the dispersion after the exclusion of the local $>3 \sigma$ outliers performed in Sect. 4.1. They are computed from the final cleaned samples of $318(\mathrm{Sgr}, \mathrm{N})$ and $417(\mathrm{M} 54)$ stars, as already reported in Sect. 3.5, above.

rows of the table). The primary bins are enclosed by the vertical lines. In each bin we computed the average $V_{r}$ and the velocity dispersion $\sigma$, with their uncertainties (derived with the "jackknife" method, as above). An iterative $3 \sigma$ clipping algorithm was applied bin by bin. As we proceeded from the innermost bin to the outer ones, any star rejected in a given bin by the clipping algorithm was excluded from the sample. The eight rejected stars are clearly indicated in the plot.

The derived velocity dispersion profile is reported in the lower panel of Fig. 11 (large filled pentagons, primary set, small filled pentagons, secondary set) and in Tab. 6. The table reports also an alternative estimate of $\sigma$ obtained with the Gaussian maximum-likelihood method described by Walker et al. (2006), essentially equivalent to that adopted by Martin et al. (2007). It is remarkable that in all cases the estimates obtained with the two methods differ by much less than the reported uncertainties (in all cases by $\leq 0.3 \mathrm{~km} / \mathrm{s}$ ). The profile is complemented with the central estimate obtained by Illingworth (1976) from integrated spectroscopy of the cluster core ${ }^{7}$. The profile shows a steep decrease from $\sigma=14.2 \mathrm{~km} / \mathrm{s}$ at the center to $\sigma \simeq 5.0 \mathrm{~km} / \mathrm{s}$ at $r=3.5^{\prime}$. Then it begins to grow gently up to $\sigma \simeq 9 \mathrm{~km} / \mathrm{s}$ in the last bin $\left(r \simeq 7^{\prime}\right)$.

Fig. 12 shows the velocity distribution and dispersion profile for the Sgr,N sample, with the same arrangement as in Fig. 11, above. The absence of any obvious trend

7 Here we adopt the estimate of central velocity dispersion $\sigma_{0}=14.2 \mathrm{~km} / \mathrm{s}$ obtained by Illingworth by correcting the value he measured from integrated long-slit spectroscopy of the cluster center.
TABLE 6

VELOCITY DISPERSION PROFILE FOR M54.

\begin{tabular}{cccccccc}
\hline $\begin{array}{c}r_{i} \\
\operatorname{arcmin}\end{array}$ & $\begin{array}{c}r_{f} \\
\operatorname{arcmin}\end{array}$ & $\begin{array}{c}\mathrm{r}_{m} \\
\operatorname{arcmin}\end{array}$ & $\begin{array}{c}\sigma \\
\mathrm{km} / \mathrm{s}\end{array}$ & $\begin{array}{c}\mathrm{e}_{\sigma} \\
\mathrm{km} / \mathrm{s}\end{array}$ & $\begin{array}{c}\sigma_{m l} \\
\mathrm{~km} / \mathrm{s}\end{array}$ & $\begin{array}{c}\mathrm{e}_{\sigma} \\
\mathrm{km} / \mathrm{s}\end{array}$ & $\mathrm{N}$ \\
\hline 0.1 & 1.0 & 0.68 & 10.2 & 0.9 & 10.1 & 1.0 & 95 \\
0.5 & 1.5 & 0.96 & 10.2 & 0.7 & 10.1 & 1.1 & 131 \\
1.0 & 2.0 & 1.50 & 8.6 & 0.5 & 8.5 & 1.2 & 107 \\
1.5 & 2.5 & 1.98 & 7.4 & 0.4 & 7.2 & 1.4 & 101 \\
2.0 & 3.0 & 2.47 & 7.4 & 0.5 & 7.3 & 1.3 & 86 \\
2.5 & 3.5 & 2.95 & 6.2 & 0.6 & 6.1 & 1.3 & 66 \\
3.0 & 4.0 & 3.38 & 5.3 & 0.7 & 5.1 & 1.2 & 42 \\
3.5 & 5.0 & 4.29 & 6.3 & 0.5 & 6.2 & 1.1 & 49 \\
4.0 & 6.0 & 4.83 & 6.7 & 0.5 & 6.6 & 1.1 & 53 \\
5.0 & 7.0 & 5.89 & 7.0 & 0.9 & 6.9 & 0.9 & 36 \\
6.0 & 9.0 & 6.87 & 8.8 & 1.5 & 8.6 & 0.7 & 34 \\
\hline
\end{tabular}

NOTE. $-r_{i}$ and $r_{f}$ are the limits of the bins, $r_{m}$ is the radius of the middle of the bins. $\mathrm{N}$ is the number of stars in the bin, after the rejection of $3 \sigma$ outliers. $\sigma_{m l}$ is the velocity dispersion estimated with a Gaussian maximum likelihood method as done in Walker et al. (2006).

TABLE 7

Velocity dispersion PROFILE FOR SGR,N.

\begin{tabular}{cccccccc}
\hline $\begin{array}{c}r_{i} \\
\operatorname{arcmin}\end{array}$ & $\begin{array}{c}r_{f} \\
\operatorname{arcmin}\end{array}$ & $\begin{array}{c}\mathrm{r}_{m} \\
\operatorname{arcmin}\end{array}$ & $\begin{array}{c}\sigma \\
\mathrm{km} / \mathrm{s}\end{array}$ & $\begin{array}{c}\mathrm{e}_{\sigma} \\
\mathrm{km} / \mathrm{s}\end{array}$ & $\begin{array}{c}\sigma_{m l} \\
\mathrm{~km} / \mathrm{s}\end{array}$ & $\begin{array}{c}\mathrm{e}_{\sigma} \\
\mathrm{km} / \mathrm{s}\end{array}$ & $\mathrm{N}$ \\
\hline 0.8 & 2.0 & 1.41 & 9.1 & 0.9 & 8.9 & 0.7 & 41 \\
2.0 & 3.0 & 2.55 & 8.8 & 1.2 & 8.6 & 0.7 & 38 \\
3.0 & 4.0 & 3.54 & 10.2 & 0.9 & 10.0 & 0.7 & 56 \\
4.0 & 5.0 & 4.47 & 8.8 & 1.0 & 8.5 & 0.9 & 43 \\
5.0 & 6.0 & 5.46 & 9.6 & 1.1 & 9.3 & 0.7 & 40 \\
6.0 & 7.0 & 6.52 & 8.7 & 0.9 & 8.5 & 0.7 & 43 \\
7.0 & 9.0 & 7.69 & 10.3 & 1.1 & 10.1 & 0.7 & 56 \\
\hline
\end{tabular}

NотE. $-r_{i}$ and $r_{f}$ are the limits of the bins, $r_{m}$ is the radius of the middle of the bins. $\mathrm{N}$ is the number of stars in the bin, after the rejection of $3 \sigma$ outliers. $\sigma_{m l}$ is the velocity dispersion estimated with a Gaussian maximum likelihood method as done in Walker et al. (2006).

of velocity dispersion with radius allowed us to adopt a single set of bins of nearly uniform size. The dispersion in each bin has been estimated exactly as in the case of M54, described above. The two innermost stars of the sample (crosses) have been excluded from the analysis as they lie in a too poorly sampled radial region $\left(r<0.8^{\prime}\right)$ and may affect the computation of the mean radius of the first bin. However, the inclusion of these stars in the innermost bin changes the velocity dispersion estimate 


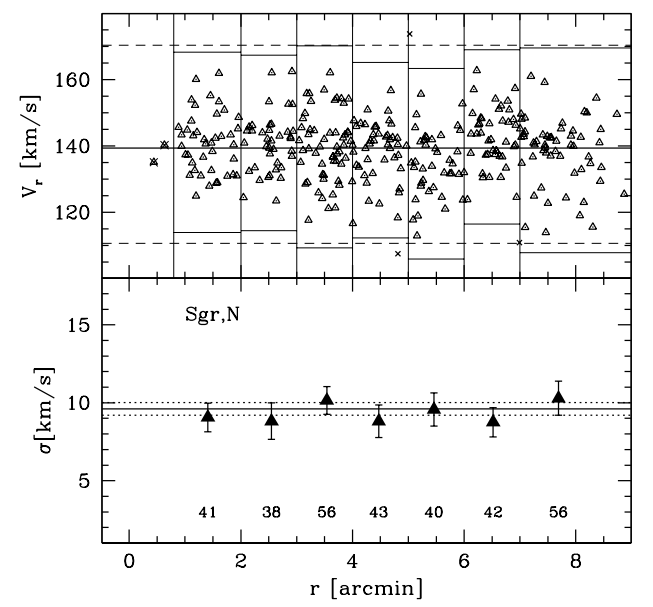

FIG. 12.- The same as Fig. 11 for the Sgr,N sample (triangles replaces pentagons here). The two innermost point are rejected for reasons of uniformity of the sample. In the lower panel we also report the velocity dispersion of the whole sample as a continuous line, \pm the associated error (dotted lines). As $\sigma$ does not appear to change with radius we use only the reported seven independent bins.

by $\simeq 0.2 \mathrm{~km} / \mathrm{s}$.

It is quite obvious from the inspection of Fig. 12 that the velocity dispersion profile of $\mathrm{Sgr}, \mathrm{N}$ is completely flat over the whole radial range explored by our data. The lower panel shows that the velocity dispersion of each radial bin is in good agreement with the velocity dispersion of the whole sample after the clipping of $3 \sigma$ outliers, $\sigma=9.6 \pm 0.4 \mathrm{~km} / \mathrm{s}$. The maximum observed difference among bins is $1.6 \pm 1.4 \mathrm{~km} / \mathrm{s}$; the maximum difference between the dispersion in a given bin and the dispersion of the whole sample is $-0.9 \pm 1.0$. Possibly, there may even be a weak tendency toward lower velocity dispersions in the inner regions: in the range $0.0^{\prime} \leq r<5.0^{\prime}$ we find $\sigma=9.2 \pm 0.5 \mathrm{~km} / \mathrm{s}$ (180 stars), while in the range $5.0^{\prime} \leq r<9.0^{\prime}$ we find $\sigma=10.1 \pm 0.6 \mathrm{~km} / \mathrm{s}$ (138 stars); however this difference is clearly not statistically significant (see Geha, Guhathakurta \& van der Marel 2002; Valluri et al. 2005, for some example of the variety of velocity dispersion profiles observed in $\mathrm{dE}, \mathrm{N}$ ).

It is rather clear that the nucleus of Sgr and the M54 cluster have different velocity dispersion profiles. M54 displays the typical behavior of a globular cluster, i.e. the system becomes increasingly kinematically hot toward its central regions while Sgr,N has a uniformly flat profile, though the surface brightness profiles of the two systems are broadly similar. Fig. 13 shows the comparison between the profiles in deeper detail. The inner growth of $\sigma$ of M54 stars makes the two profiles overlap at $r \sim 1.5^{\prime}$; the innermost two points of the M54 profile are hotter than any Sgr,N point. The outermost point of the cluster profile does match the dispersion of Sgr,N: the velocity dispersion estimate here comes from the least populated bin as well as the most (possibly) prone to contamination by old and metal-poor stars belonging to Sgr (see below for a more detailed discussion). These are the reasons why the comparison between the two samples taken as a whole does not reveal any striking difference. However, in the intermediate radial range the M54 population is
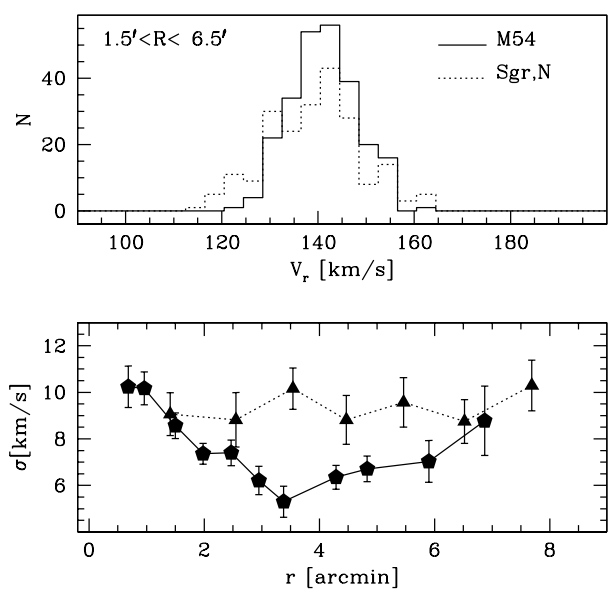

FIG. 13.- Upper panel: Velocity distribution of the M54 and Sgr, $\mathrm{N}$ samples in the radial range in which the difference in their $\sigma$ reaches the maximum statistical significance, according to the F test (see Tab. 9] Lower panel: Comparison of the velocity dispersion profiles of M54 (continuous line and pentagons) and Sgr,N (dotted line and triangles).

significantly colder than that of Sgr, $\mathrm{N}$ and the statistical significance of this result is very strong.

Tab. 8 reports the results of non-parametric Kolmogorov-Smirnov tests performed on different radial ranges. In all the considered ranges the probability that the two samples are drawn from the same parent population is smaller than $2 \%$ and it is smaller than $0.2 \%$ in the $1.5^{\prime} \leq r<6.5^{\prime}$ range. In the present case, however, the $\mathrm{F}$ statistic is the most appropriate mean to compare statistically the two samples $\left(F=\sigma_{S g r}^{2} / \sigma_{M 54}^{2}\right.$, see Brandt 1970, and references therein), as the corresponding $\mathrm{F}$ test evaluates the probability that two samples of given $\mathrm{F}$ value are extracted from Gaussian distributions having the same $\sigma$. Tab. 9 shows that in our case this probability is lower than $0.3 \%$ in all the considered radial range and it is as small as $<0.01 \%$ in several intermediate ranges, including $1.5^{\prime} \leq r<6.5^{\prime}$. While such a large quantitative difference may not be so obviously apparent from the dispersion profile, it is clear at a first glance when looking at the comparisons in the $\mathrm{X}-V_{r}$ and in the $\mathrm{R}-V_{r}$ planes shown in Fig. 14.

Hence, the direct comparison of the velocity distributions of the two samples clearly establishes that the two populations have very different kinematic properties, indicating that the motion of M54 and Sgr, $N$ stars is driven by different gravitational equilibria.

Moreover, Gilmore et al. (2007) clearly states that a velocity dispersion profile monotonically declining from the center outward "...is an unavoidable requirement for any mass - follows - light system...". In the present case, this means that while M54 behaves like an ordinary mass - follows - light "purely baryonic" self-gravitating star cluster (at least in its innermost region, containing most of its light/mass; see also Fig. 14 and Sect. 4.2, below), the flat velocity dispersion profile of Sgr, $N$ implies that the mass distribution driving the kinematics of the nucleus is significantly different from the distribution of the stars. In principle, a radial gradient in the velocity 

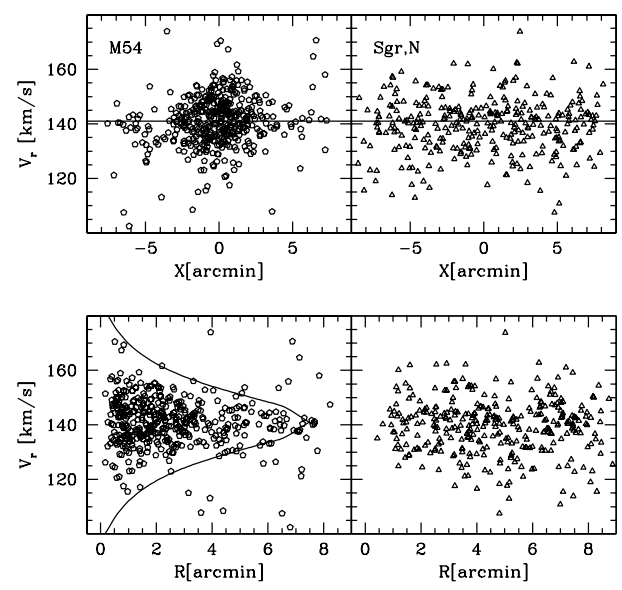

FIG. 14.- Direct comparison of the velocity and spatial distributions of M54 and Sgr,N. Upper panels: $V_{r}$ as a function of X for the M54 (left panel) and for the Sgr,N (right panel) samples. Lower panels: $V_{r}$ as a function of $\mathrm{R}$ for the same samples. The curves in the lower left panel are the $\pm 3 \sigma$ profiles the best-fit King model for M54 (Trager. King \& Diorgovski 1995), approximately representing the envelope of the allowed velocities for bound members of M54.

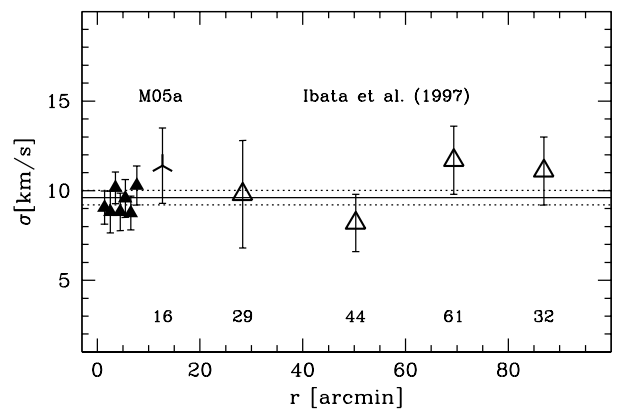

Fig. 15. - Velocity dispersion profile of the innermost $\sim 100^{\prime}$ of the Sgr galaxy. Filled triangles are the $r<10^{\prime}$ estimates obtained here, the skeletal symbol is from the M05a stars having $[\mathrm{Fe} / \mathrm{H}]<$ -1.0 (to avoid possible M54 members), open triangles are from Table $2 \mathrm{~b}$ of Ibata et al. (1997) (CTIO-ARGUS radial velocities). The horizontal lines are the same as in Fig. 12 Note that this composite profile covers less than half of the core radius of the Sgr galaxy $\left(r_{c} \simeq 220^{\prime}\right.$ Majewski et al. 2003).

anisotropy affecting only one of the two systems may be invoked to explane the observed difference in the velocity dispersion profiles. As this would imply a correlation of the anisotropy variation with metallicity, we regard this hypotesis as rather "ad-hoc" and we don't discuss it anymore, in the following. We will re-consider the case in more detail in a future contribution (Ibata et al., in preparation).

Finally, the velocity dispersion profile of Sgr,N does not differ significantly from the overall profile of the
TABLE 8

KS test. Probability that the SGR,N And the M54 SAmples ARE DRAWN FROM THE SAME PARENT POPULATION.

\begin{tabular}{cccccc}
\hline$r_{i}$ & $r_{f}$ & $\mathrm{~N}_{M 54}$ & $\mathrm{~N}_{S g r, N}$ & $\mathrm{D}_{K S}$ & $\mathrm{P} \%$ \\
\hline 0.0 & 9.0 & 417 & 318 & 1.649 & 0.87 \\
0.8 & 8.0 & 353 & 300 & 1.531 & 1.84 \\
2.0 & 7.0 & 199 & 219 & 1.624 & 1.02 \\
2.0 & 6.0 & 181 & 177 & 1.698 & 0.63 \\
1.5 & 6.5 & 247 & 213 & 1.817 & 0.13 \\
\hline
\end{tabular}

Note. - KS $=$ Kolmogorov-Smirnov. $r_{i}$ and $r_{f}$ are the limits of the considered radial ranges, $\mathrm{N}_{\text {sample }}$ is the number of stars of the sample in the considered radial range. $3 \sigma$ outliers have been excluded from the samples.

\section{TABLE 9}

F test. Probability that the Sgr, N and the M54 Samples ARE DRAWN FROM GAUSSIAN DISTRIBUTIONS HAVING THE SAME $\sigma$.

\begin{tabular}{cccccc}
\hline$r_{i}$ & $r_{f}$ & $\mathrm{~N}_{M 54}$ & $\mathrm{~N}_{S g r, N}$ & $\mathrm{~F}$ & $\mathrm{P} \%$ \\
\hline 0.0 & 9.0 & 417 & 318 & 1.345 & 0.23 \\
0.8 & 8.0 & 353 & 300 & 1.448 & 0.04 \\
2.0 & 7.0 & 199 & 219 & 2.010 & $<0.01$ \\
2.0 & 6.0 & 181 & 177 & 2.019 & $<0.01$ \\
1.5 & 6.5 & 247 & 213 & 1.978 & $<0.01$ \\
2.5 & 3.5 & 65 & 51 & 2.546 & 0.02 \\
1.5 & 3.5 & 166 & 80 & 1.862 & 0.04 \\
\hline
\end{tabular}

Note. $-r_{i}$ and $r_{f}$ are the limits of the considered radial ranges, $\mathrm{N}_{\text {sample }}$ is the number of stars of the sample in the considered radial range. $3 \sigma$ outliers have been excluded from the samples. $\mathrm{F}$ is computed as $F=\sigma_{S g r, N}^{2} / \sigma_{M 54}^{2}$.

Sgr galaxy, that is found to be flat out to large radii (Ibata et al. 1997), as typical of dwarf spheroidals (see Walker et al. 2006; Muñoz. Majewski \& Johnston 2007, and references therein). In Fig. 15] we have combined the inner $0.8^{\prime} \leq r \leq 9^{\prime}$ profile obtained here, with estimates of the dispersion at different radii out to $r \simeq 100^{\prime}$ obtained from publicly available data from the literature (M05a, Ibata et al. 1997) in the same way as done for M54 and Sgr,N, above. Note that $r \simeq 100^{\prime}$ is less than half of the core radius of the whole Sgr galaxy (Majewski et al. 2003). All the observations are consistent with a constant $\sigma$ in the considered range and, in particular, there is no apparent transition in the profile at the onset of the nuclear overdensity of stars $\left(r \leq 10^{\prime}\right)$. This fact strongly suggests that the inner kinematics of Sgr, including its nucleus, are dominated by the potential set by a distribution of unseen (Dark) matter, not by M54 as in the scenario (b) described in Sect. 1., above.

\subsection{Comparison with models}

In Fig. 16] the observed velocity dispersion profile of M54 is compared with the theoretical profiles of singlemass isotropic King (1966) models that have been proposed by various authors as best-fits to the surface brightness profile of the cluster (see Tab. 4). The models proposed by Trager. King \& Diorgovski (1995, continuous line) and by McLaughlin \& van der Marel (2005, dashed line) provide a reasonable description of the data out to $r \lesssim 4^{\prime}$, while there is a clear tendency of the data to be hotter than the models for $r>4^{\prime}$. The outermost observed point, at $r \simeq 7^{\prime} \lesssim r_{t}$, displays a velocity dispersion significantly higher than what is predicted even by the hottest model $\left[\sigma_{o b s}-\sigma_{K M}\right]_{r \sim 7^{\prime}}=5.8 \pm 2.0 \mathrm{~km} / \mathrm{s}$. 


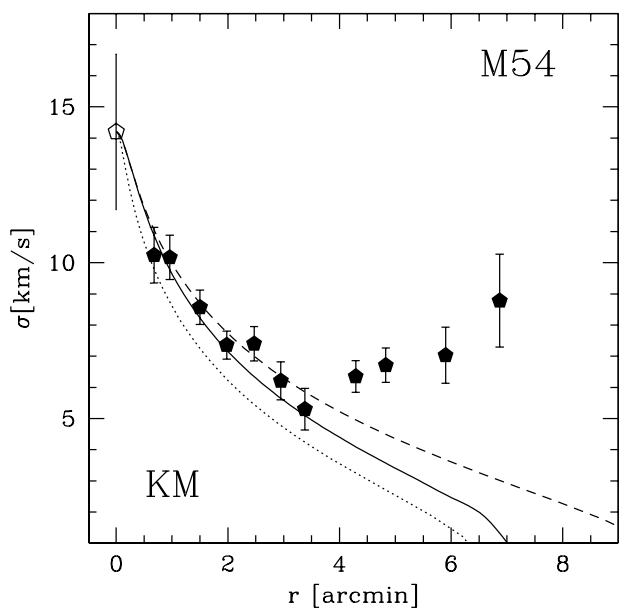

FIG. 16.- Comparison of the observed velocity dispersion profile of M54 with King models that best fit the Surface Brightness profile of the cluster, as proposed by various authors (see Tab. 4), and normalized to $\sigma_{0}=14.2 \mathrm{~km} / \mathrm{s}$. Continuous line: $r_{c}=0.11^{\prime}$ and $C=1.85$, from Trager, King \& Diorgovski (1995). Dotted line: $r_{c}=0.053^{\prime}$ from Novola \& Gebhardt $(2006)$ and $C=2.10$, obtained by adopting the estimate of the tidal radius from Trager et al., since it is not provided by Noyola \& Gebhardt. Dashed line: $r_{c}=0.09^{\prime}$ and $C=2.05$, from McLaughlin \& van der Marel (2005).

There are two possible explanations for this unexpected rise of the velocity dispersion curve near the tidal radius of the cluster.

First, while our selection criteria prevents any contamination of the Sgr,N sample by M54 stars, since cluster stars with $[\mathrm{Fe} / \mathrm{H}] \geq-0.8$ simply do not exist, it is well known that the Sgr galaxy does host a minority of stars with $[\mathrm{Fe} / \mathrm{H}] \leq-1.0(\lesssim 12 \%$, Monaco et al. 2003), hence some degree of contamination of the M54 sample by Sgr stars is expected (see Sect. 3.1) and the less populated outer bins of the M54 profile are clearly the most sensitive to the effect of the inclusion of contaminants. Rough estimates indicate that the contamination by metal poor Sgr stars should amount to less than $3 \%$ in the innermost $2^{\prime}$, less than $10 \%$ for $2^{\prime} \leq r<4^{\prime}$, but it can raise to $\lesssim 20 \%$ for $4^{\prime} \leq r<6^{\prime}$ and be even larger for $r>6^{\prime}$. So, contamination by metal-poor Sgr stars seems a viable explanation for the raise of the dispersion profile of M54 such that it becomes similar to that of the Sgr,N sample in the outermost, and presumably most contaminated, bin.

Alternatively, it may be conceived that, during its spiraling toward the center of Sgr,N (see Sect. 5.2, below), M54 suffered some tidal harassment from the host galaxy (Sgr). In this case, some stars in the last bins would have been stripped from the outskirts of the cluster and heated up to the same dispersion of the surrounding field (see Muñoz. Majewski \& Johnston 2007, and references therein). In the lower left panel of Fig. 14 the $\pm 3 \sigma$ dispersion profiles of the best-fitting King model for M54 (Trager, King \& Djorgovski 1995) are superposed on the M54 sample in the R vs. $V_{r}$ plane: the good match between the shape of the bulk of the observed distribution and the model profile out to the tidal radius strongly suggests that most of the velocity outliers at large radii

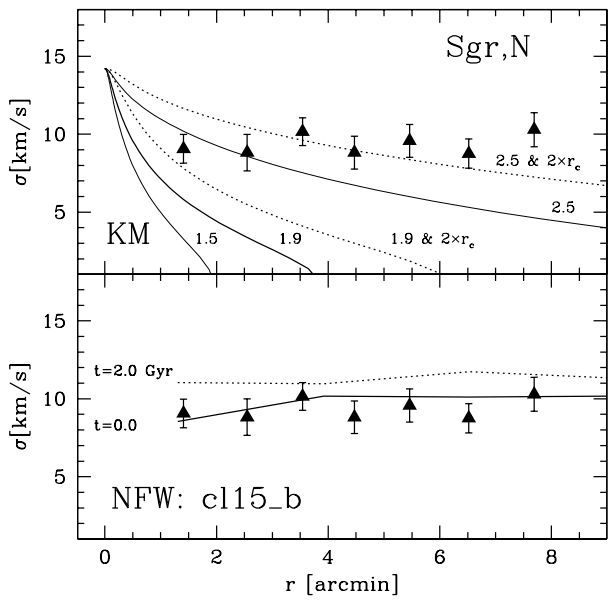

FIG. 17.- Comparison of the observed velocity dispersion profile of Sgr, $\mathrm{N}$ with theoretical models. Upper panel: comparison with King models of $r_{c}=0.05^{\prime}$ (best fit value) and various $\mathrm{C}$ (thin continuous lines labeled with their $\mathrm{C}$ value), normalized at the central velocity dispersion of M54 $\left(\sigma_{0}=14.2 \mathrm{~km} / \mathrm{s}\right)$. The $C \sim 1.9$ case corresponds to the model that best-fits the observed SB profile and is plotted with a thicker line. The dotted curves show the effect on the profile of the assumption of $r_{c}$ and $r_{t}$ values much larger than the best fit value (of a factor $\times 2$ ). Lower panel: the observed velocity dispersion profile is compared with the profile of the NFW model adopted in the N-body simulation cl15_b (see Tab. 10). The profiles at the beginning (continuous line) and at the end (dotted line) of the simulation are shown.

are likely not gravitationally bound to the cluster, independent of their origin (unrelated metal poor Sgr stars or former M54 members that have been tidally stripped).

While we consider the "contamination" hypothesis as more likely, the "tidal" hypothesis is a very fascinating possibility and it cannot be dismissed with the data we have presently in hand (see also Sect. 4.3, below).

Turning back to the inner regions of the cluster, the main conclusion we can draw here is that the observed velocity dispersion profile is consistent with the expected kinematics of the King models that best fit the light distribution of the cluster. Hence, M54 has the kinematics of an ordinary self-gravitating globular cluster, at least in its innermost $\sim 25 \mathrm{pc}$, enclosing more than $90 \%$ of the cluster light/mass.

\subsubsection{The case of $S g r, N$}

In Fig. 17 the observed velocity dispersion profile of $\mathrm{Sgr}, \mathrm{N}$ is compared with various theoretical models. In the upper panel we compare King's models with $r_{c}=$ $0.05^{\prime}$ and various values of the concentration parameter $\mathrm{C}$ (the best-fit model for the SB profile corresponds to $\mathrm{C}=1.9$ ). All the theoretical profiles have been normalized at the central velocity dispersion of M54: this is a quite arbitrary choice, the family of profiles can be shifted up and down according to the preference of the reader. It is clear that, independent of the adopted normalization, none of the plotted profiles provides a satisfactory match to the observations. The dotted profiles, reported here for comparison, show the effects of factor $2 \times$ changes in the adopted values of $r_{c}$ and $r_{t}$. Even if such large values were compatible with the observations presented in Sect. 2, above (and they are not), the corresponding 
profiles remain unable to reproduce the observed dispersions.

One could be tempted to suggest that any King model with sufficiently large scale to fit the whole surface profile of the Sgr galaxy (as the $\mathrm{C}=0.90, r_{c}=224^{\prime}$ model by Majewski et al. 2003) would have a nearly flat dispersion profile on the relatively small radial range studied here $\left(r \leq 9^{\prime}\right)$, thus providing a reasonable fit to the observed profile. This is true, but these models would completely fail in reproducing the observed Surface Brightness profile in the same range by more than $10 \mathrm{mag} / \operatorname{arcsec}^{2}$ (see Fig. 2, above), since they lack any nuclear overdensity at their center.

Hence, contrary to the case of M54, King models that fit the surface brightness profile of Sgr,N appear unable to reproduce its kinematics: this further supports the conclusion that M54 and Sgr,N are systems of very different nature ${ }^{8}$. In particular, as already noted, mass follows -light models are incompatible with the observed velocity dispersion profile of Sgr,N (Gilmore et al.|2007).

In the lower panel of Fig. [17 the observed profile is compared to those obtained from the N-body realization of a Navarro, Frenk \& White (1996, hereafter NFW) model of the suite that is described in Sect. 5, below (model NFW3, Tab. 10). The continuous line is the inner velocity dispersion profile of the NFW3 model at the beginning of the simulation; the dotted line is the profile at the end of the simulation, i.e. after the complete orbital decay of a model of M54 that is launched in orbit within the NFW3 halo. It is interesting to note that the theoretical profiles are fairly flat in the considered radial range, in good agreement with the data. It may be conceived that the (baryonic) nucleus of Sgr lies within the inner cusp of the NFW halo of dark matter that is presumed to embed the Sgr galaxy (Ibata \& Lewis 1998; Majewski et al. 2003, and references therein). The potential in this inner region would be dominated by the DM cusp that will impose the flat dispersion curve to the observed Sgr, N stars. It does not seem necessary to assume a strict correlation between the typical size and/or density profile of the DM cusp/core and those of the embedded stellar nucleus: the DM overdensity would have simply provided the "local" minimum of the overall potential well to "attract" the largest density of the infalling gas that formed Sgr,N. It may be interesting to note that the NFW3 model, whose velocity dispersion profile is shown in the lower panel of Fig. refsisag, encloses a mass of $3.2 \times 10^{6} M_{\odot}$ within the range of projected radii covered by our data $\left(r_{p} \simeq 70\right.$ pc), and that the region in which its projected density profile is well fitted by a power-law, i.e. the inner cusp, has a size of $r_{p} \lesssim 100 \mathrm{pc}$, similar to Sgr,N.

Unfortunately, we have no conclusive observational evidence in support of the above scenario. Note also that a DM halo with a core much larger than the nuclear scale would also display a flat dispersion curve, in this region (as discussed above for the case of the $\mathrm{C}=0.90, r_{c}=224^{\prime}$ King model); it is just the small-scale inner cusp as a possible seed for the formation of a barionic nucleus that can make the NFW option slightly more attractive.

\footnotetext{
${ }^{8}$ We stress here that we are not attaching any particular physical meaning to King models while fitting Sgr,N profiles. This is just another way to put in evidence the differences between Sgr,N and M54 and a convenient reference model to parametrize the observed SB profile (Muñoz, Majewski \& Johnston 2007).
}

A flat velocity dispersion profile may arise as a consequence of strong tidal disturbance, in mass-follow-light models of dwarf galaxies (Muñoz, Ma jewski \& Johnston 2007). While Sgr is clearly in course of tidal disruption, here we are dealing with the kinematics of the innermost $\simeq 70$ pc of the galaxy, that should (reasonably) be considered as untouched by the Galactic tides, since the tidal radius of Sgr is as large as a few thousands of parsecs (the formal King's major axis limiting radius is $r_{t} \gtrsim 10 \mathrm{kpc}$, according to Majewski et al. 2003).

Finally, it may appear as a curious coincidence that M54 and Sgr, $N$ have similar spatial scales, if they have independent origin. In this sense we can only note that the distributions of sizes and luminosities of globular clusters and stellar galactic nuclei largely overlap, so the observed similarity may not be particularly odd (see Federici et al. 2007; Böker 2007). Moreover, it should be noted that the core and half-light radii of M54 are $\sim 2$ times larger than those of Sgr,N, clearly a non-negligible difference. Finally, while the best fitting King models have essentially the same $\mathrm{C}$ parameter, the two observed profiles appear to have significantly different slopes at large radii, where the profile of Sgr,N departs from the King model.

\subsection{Rotation}

We searched for signal of rotation in the two samples, also taking into account the correction for perspective rotation, according to van de Ven et al. (2006) and taking the proper motion of Sgr/M54 from Dinescu et al. $(2005)$; in the present case the absolute value of the maximum correction is $\leq 1.0 \mathrm{~km} / \mathrm{s}$. Given a rotation of the coordinate axes by an angle $\theta$

$$
\begin{aligned}
& \eta=X \cos (\theta)-Y \sin (\theta) \\
& \chi=X \sin (\theta)+Y \cos (\theta)
\end{aligned}
$$

we tried all the possible values of $\theta$ in steps of $1^{\circ}$. For each adopted $\theta$ we computed the median velocity of stars with negative and positive $\eta$. The difference between the two median velocities is a robust measure of the amplitude of any systemic motion around an axis tilted by $\theta$ degrees from the $\chi$ axis, i.e. of the rotation of stars about this axis.

In some cases, ordered patterns of $V_{r}$ as a function of $\eta$ emerged (in particular for the M54 sample), but their amplitude was quite weak, typically lower than 2.0 $\mathrm{km} / \mathrm{s}$. While we can clearly exclude the presence of rotation signals stronger than this, we cannot exclude that such weak patterns are in fact real. In Fig. 18 we show the best case for the M54 sample compared with the end product of one of the N-body simulations that are described in Sect. 5, below. We refrain from attaching any significance to such a weak pattern, in particular as it has an amplitude similar to the typical uncertainty of the individual velocity measures and as it is much weaker than the typical velocity dispersion of the sample. We just want to draw the attention of the reader to the fact that the orbital decay of a massive cluster within the parent dwarf galaxy seems to naturally produce these kinds of weak velocity gradients in the inner part of the sinking cluster, at the end of the decay process (see Muñoz, Majewski \& Johnston 2007, for a deeper discussion of the effects of tidal disruption). However, it is quite clear that the detection at $r \gtrsim 10^{\prime}$ of stars 

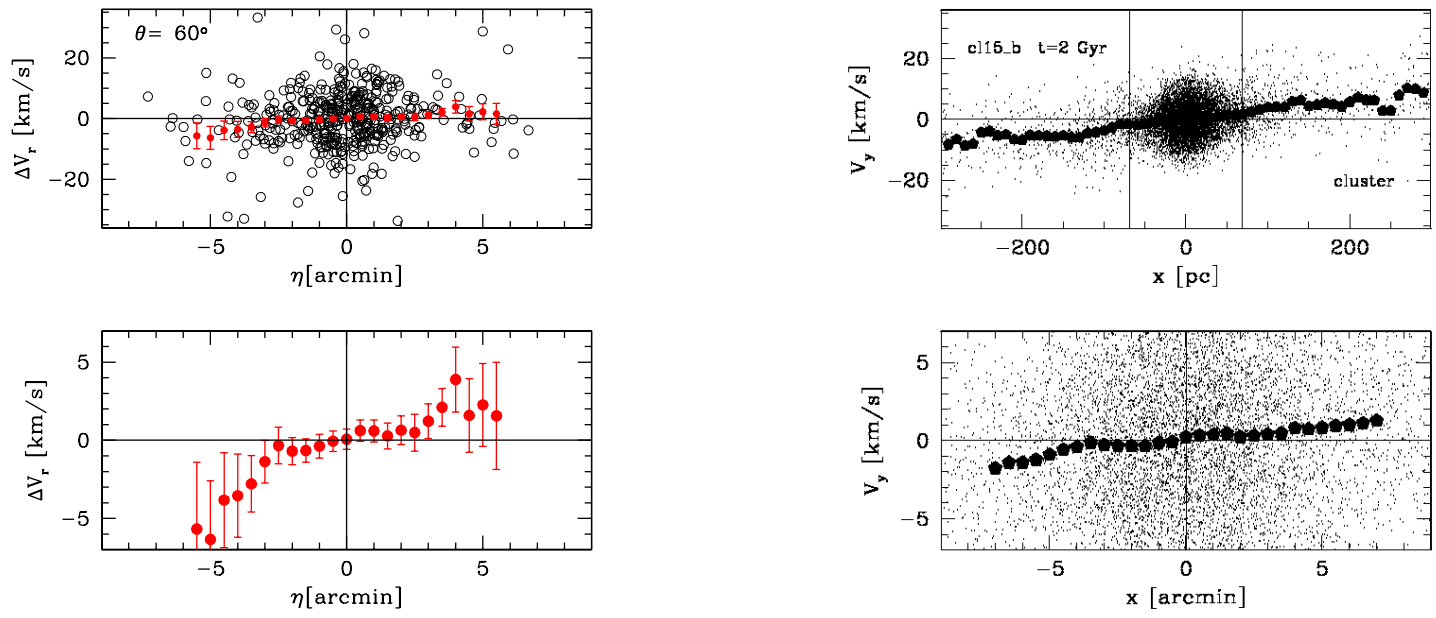

FIG. 18. - Left Panels: Rotation curve of M54 assuming a rotation axis tilted by $\theta=60^{\circ}$ East of North. $\Delta V_{r}$ is the difference between the observed radial velocity and the global mean systemic velocity of Sgr, $\mathrm{N}\left(\left\langle V_{r}\right\rangle=\mathrm{km} / \mathrm{s}\right)$. Upper panel: individual stars. Lower panel: running mean with bin width of $2^{\prime}$ and step of 0.5 . Right Panels: Rotation in the remnant of the model of M54 at the end of the simulation cl15_b. The coordinate system is chosen such that x,z projects on the plane of the sky while y is along the line of sight and the orbit of the cluster is in the $\mathrm{x}, \mathrm{y}$ plane. Upper panel: l.o.s. velocity as a function of $\mathrm{x}$ for the innermost $300 \mathrm{pc}$. The vertical thin lines enclose a region of the same dimension as the one studied here; the large dots are the running mean of the velocity computed with the same bin width and step as in the real case. Note the tidal tails emerging from the main body of the remnant at $x \sim \pm 50$ pc. Lower panel: zoomed view of the inner region of the plot. In this case $\mathrm{x}$ has been converted in arcmin by placing the remnant at the same distance as the real cluster. The diagram has the same scale as the lower left panel for an easy comparison.

having metallicity compatible with M54, possibly lower velocity dispersion than the surrounding metal-rich $\mathrm{Sgr}$ population, and, above all, having significant differences in mean systemic velocity compatible with a tidally induced rotation like that shown in the upper right panel of Fig. 18, would be the final "smoking-gun" of the orbital decay of M54 to its current position. On the other hand the failure to find such a component would not be sufficient to rule out the hypothesis as, for instance, the density of stars in the tidal tails is expected to be very low and the "rotation" signal may be greatly weakened by unfavorable orientations of the orbital plane with respect to the line of sight (Muñoz, Majewski \& Johnston 2007).

\section{THE KINEMATICS OF M54 AND SGR,N: N-BODY SIMULATIONS}

M05a used simple analytical formulae to verify the plausibility of the hypothesis that the "exact" coincidence between the positions of M54 and Sgr,N is due to the cluster progressively spiraling into the center of density of the Sgr galaxy due to dynamical friction. They found that the observed status of the globular cluster system of Sgr is in full agreement with this hypothesis. The orbit of the massive M54 cluster is expected to decay completely within one Hubble time if it is born within 3 core radii $(\sim 5 \mathrm{kpc})$ from the center of Sgr, while the other, much less massive Sgr clusters (Ter 7, Ter 8, Arp 2) would be essentially unaffected by dynamical friction if they were born outside 1 core radius. While promising, this first result demands further investigation. One very interesting question, for instance, is: does the actual orbital decay of M54 under realistic conditions lead to such a nearly perfect coincidence between M54 and Sgr, N as observed? To answer questions like this we performed the suite of N-body simulations that is described in de- tail below. While examining the results of our N-body experiments it should be carefully considered that:

- The main purpose of our simulations is to study the effect of dynamical friction in the specific case of M54 orbiting within the Sgr galaxy, with particular focus on the final status of the system. Detailed and more general theoretical analyses of the effects of dynamical friction on globular clusters within a dwarf galaxy can be found in Oh, Lin \& Richer (2000), Read et al. (2006), Fujii, Funato \& Makino (2006), Miocchi et al. (2006), and Sánchez-Salchedo, Reves-Iturbide \& Hernandez (2006), and references therein.

- We do not intend our simulations to be exhaustive of all the possible cases. This would be prohibitive since, for example, we do not even know what is the total mass of the bound part of the Sgr galaxy (see Majewski et al. 2003, and references therein). Our aim is to verify if a broadly realistic case can produce the final outcome we actually observe, i.e. a cluster that has nearly exactly the same position and velocity as the nucleus of its host galaxy.

- Here we study the case of a massive cluster orbiting within a model of the Sgr galaxy which evolves in isolation. We verified that this assumption does not seriously affect our main results. We replicated some of our experiments launching the Sgr model into a realistic (but static) Galactic potential (model 2d of Dehnen \& Binney 1998): we found that if M54 was not lost into the tidal tails of the disrupting Sgr galaxy (as it happened for some Sgr clusters, see Bellazzini, Ferraro \& Ibata 2003a), it plunged to the center of Sgr approximately on the same timescales as the isolated models. 
- A detailed comparison with the existing literature on dynamical friction would be clearly beyond the scope of the present paper (see, for instance Colpi, Maver \& Governato 1999, and references therein). In the present context, suffice to say that our results are in reasonably good agreement with the analytic estimates by M05a as well as with the results by Oh. Lin \& Richer (2000); Sánchez-Salchedo, Reves-Iturbide \& Hernandez (2006); Read et al. (2006, and references therein).

\subsection{General features of the $N$-body experiments}

The simulations were performed with falcon, a fast and momentum-conserving tree-code (Dehnen 2000, 2002), within the NEMO environment (Teuben 1995). Gravity was softened with the kernel ' $\mathrm{P}_{2}$ ' (Dehnen 2000), with a softening length of $3 \mathrm{pc}$ (except for two special cases, bh_15p and bh_02a, discussed in Sect. 5.2, below). The minimum time-step was $1.9 \times 10^{-6} \mathrm{Gyr}$, and the tolerance parameter $\theta=0.6$.

To model the Sgr galaxy we adopted a truncated Navarro, Frenk \& White (1996, hereafter NFW) model of the form:

$$
\rho(r) \propto \frac{\operatorname{sech}(r / b)}{r(r+a)^{2}} .
$$

For the model NFW1, the parameters $a=0.1 \mathrm{kpc}$, $b=2.5 \mathrm{kpc}$, were selected, which together with a maximum circular speed of $V_{c}=30 \mathrm{~km} / \mathrm{s}$, gives a total mass of $M_{T O T}=2.4 \times 10^{8} M_{\odot}$. This rather massive model was chosen to approximate to the sort of dense system suggested by Ibata \& Lewis (1998) that would not be rapidly destroyed by Galactic tides. A second, lower mass system (NFW2) with $a=0.1 \mathrm{kpc}, b=2.5 \mathrm{kpc}$ and $V_{c}=15 \mathrm{~km} / \mathrm{s}\left(M_{T O T}=6.1 \times 10^{7} M_{\odot}\right)$ was also simulated. The motivation for this second model was that the central velocity dispersion in this case should be similar to the observed dispersion of Sgr (while keeping $a$ and $b$ identical to the first model). The NFW3 with $a=0.5 \mathrm{kpc}, b=15.0 \mathrm{kpc}$ and $V_{c}=17 \mathrm{~km} / \mathrm{s}$ was chosen to have both a size and a central velocity dispersion similar to the present-day main body of Sgr, irrespective of its robustness to Galactic tides.

In all cases the NFW halos were modeled with $10^{5}$ particles. M54 was modeled as a single massive particle of $1,1.5$ or 2 million solar masses, as if it were a black hole ( $b h$ simulations, as opposed to $c l$ simulations, see Tab. 10 and Sect. 5.2, below). The particle is launched within the NFW halo from $(\mathrm{x}, \mathrm{y}, \mathrm{z})=(2,0,0) \mathrm{kpc}$ or $(4,0,0)$ $\mathrm{kpc}^{9}$ (note that the core radius of Sgr is $r_{c} \simeq 1.7 \mathrm{kpc}$ Majewski et al. 2003), with velocities in the y direction in the range $4.0 \mathrm{~km} / \mathrm{s} \leq V_{y} \leq 12.0 \mathrm{~km} / \mathrm{s}$, while $V_{x}=$ $V_{y}=0.0 \mathrm{~km} / \mathrm{s}$, hence the orbits of the M54 models lie in the $\mathrm{x}, \mathrm{y}$ plane. As a mere convention to simplify the discussion, we consider the y direction coincident with the line-of-sight between us and the center of Sgr, and the $\mathrm{x}$ and $\mathrm{z}$ directions as projected on the plane of the

9 In the coordinate system in which $(\mathrm{x}, \mathrm{y}, \mathrm{z})=(0,0,0) \mathrm{kpc}$ corresponds to the center of density of the model halo and a point having $\left(V_{x}, V_{y}, V_{z}\right)=(0,0,0) \mathrm{km} / \mathrm{s}$ is at rest with respect to the center of the system. The adopted range of initial distances from the center of the parent galaxy is typical of the other clusters residing in the main body of Sgr (Ter 7, Arp 2, Ter 8) which are located between $\sim 3 \mathrm{kpc}$ and $\sim 5 \mathrm{kpc}$ from the center of Sgr and should have orbits that are stable against dynamical friction (see M05a and below). sky. When needed we will select particles according to their projected radius $r_{p}=\sqrt{x^{2}+z^{2}}$.

The initial conditions of the various simulations are reported in Tab. 10. The simulations were usually stopped when it was clear that the orbit of the M54 particle had reached a stable configuration at the center of the host halo $\left(\mathrm{t}_{t o t}\right)$. The decay time $\left(\mathrm{t}_{d}\right)$ is defined as the epoch at which the 3-D apocentric distance of the M54 particle become $r_{\text {apo }} \leq 30 \mathrm{pc}$. Note that around this limit the adopted time step becomes too small with respect to the orbital period of the infalling massive particle, hence the evolution of the orbit cannot be followed further. To provide a quantitative idea of the evolution of the eccentricity of the orbit $\left(\epsilon=\left(r_{\text {apo }}-r_{\text {peri }}\right) /\left(r_{\text {apo }}+r_{\text {peri }}\right)\right)$ we report the eccentricity at the first $\left(\epsilon_{1}\right)$ and fifth $\left(\epsilon_{5}\right)$ pericentric passages. The evolution of the orbital radius and of the average velocity of the M54 models is shown in Fig. 19.

\subsection{The evolution of the orbit of M54 models}

From the inspection of Tab. 10 and Fig. 19 we can draw the following conclusions:

1. In spite of the wide range of initial conditions that has been explored, the orbit of all the M54 models decayed completely in less than 3 Gyr (5-15 orbits). At the end of our simulations M54 is always virtually at rest (mean velocity $\lesssim 1-2 \mathrm{~km} / \mathrm{s}$ ) at the very center of the host halo (within a few softening lengths). Hence the hypothesis that M54 reached its presently observed status by dynamical-frictiondriven orbital decay appears completely realistic and viable (within the limitations imposed by the resolution of our N-body experiments).

2. Two special simulations (bh15_p and bh02_a) have been performed at a lower resolution (softening length of $10 \mathrm{pc}$ ) as the $\mathrm{BH}$ was not expected to reach the densest regions of the host halo (NFW3). bh15_p explores the case of a M54-like point mass decaying from somehow extreme initial conditions (large distance - $X_{0}=5.0 \mathrm{kpc}$ - and high velocity $V_{Y, 0}=12.0 \mathrm{~km} / \mathrm{s}$ ). In six Gyr the orbit of the $\mathrm{BH}$ is sufficiently decayed to bring it within $2 \mathrm{kpc}$ from the center of the halo. The other simulations indicates that from this point it will require less than 3 Gyr to reach the very center of the halo. From the bh15_p simulation we can deduce that for starting distances larger than $5.0 \mathrm{Kpc}$ the times required for a complete decay begin to be comparable to the Hubble time, in good agreement with the results by M05, and also that it is quite possible that M54 have reached the very center of Sgr only in recent times, if it was born sufficiently far away from the center. The analytical computations by M05 also indicated that clusters with a mass similar to the other GC residing in the main body of Sgr $\left(\right.$ Ter $7, \operatorname{Arp} 2$, Ter $\left.8, M \leq 2 . \times 10^{4} M_{\odot}\right)$, would have infinitely long decay times (i.e. stable orbits) if they born at $r \gtrsim 1 \mathrm{kpc}$ from the center of Sgr. As the closest of these clusters lie at $\sim 2.9 \mathrm{kpc}$ from the center of Sgr M05a concluded that all of them are on stable orbits. Simulation bh02_a is intended to verify this conclusion, by letting evolve a 
TABLE 10

N-BODY SIMULATIONS: FUNDAMENTAL PARAMETERS AND RESUlTS.

\begin{tabular}{cccccccccccc}
\hline name & halo & $\begin{array}{c}\mathrm{M}_{\text {halo }} \\
M_{\odot}\end{array}$ & $\mathrm{N}_{\text {halo }}$ & $\begin{array}{c}\mathrm{M}_{\text {clus }} \\
M_{\odot}\end{array}$ & $\begin{array}{c}\mathrm{N}_{\text {clus }} \\
\text { bh2_a }\end{array}$ & $\begin{array}{c}\mathrm{X}_{0} \\
\mathrm{kpc}\end{array}$ & $\begin{array}{c}\mathrm{V}_{Y, 0} \\
\mathrm{~km} / \mathrm{s}\end{array}$ & $\begin{array}{c}\mathrm{t}_{d} \\
\mathrm{Gyr}\end{array}$ & $\begin{array}{c}\mathrm{t}_{\text {tot }} \\
\mathrm{Gyr}\end{array}$ & $\epsilon_{1}$ & $\epsilon_{5}$ \\
\hline bh2_b & NFW1 & $2.4 \times 10^{8}$ & $10^{5}$ & $2.0 \times 10^{6}$ & 1 & 2.0 & 4.0 & 0.37 & 0.84 & 0.86 & 0.67 \\
bh2_c & NFW1 & $2.4 \times 10^{8}$ & $10^{5}$ & $2.0 \times 10^{6}$ & 1 & 2.0 & 8.0 & 0.74 & 1.28 & 0.73 & 0.52 \\
bh2_d & NFW1 & $2.4 \times 10^{8}$ & $10^{5}$ & $2.0 \times 10^{6}$ & 1 & 2.0 & 12.0 & 1.28 & 1.58 & 0.56 & 0.45 \\
bh2_e & NFW1 & $2.4 \times 10^{8}$ & $10^{5}$ & $2.0 \times 10^{6}$ & 1 & 4.0 & 8.0 & 2.22 & 1.28 & 0.87 & 0.65 \\
bh1_a & NFW1 & $2.4 \times 10^{8}$ & $10^{5}$ & $1.0 \times 10^{6}$ & 1 & 2.0 & 4.0 & 0.67 & 0.74 & 0.83 & 0.72 \\
bh1_b & NFW1 & $2.4 \times 10^{8}$ & $10^{5}$ & $1.0 \times 10^{6}$ & 1 & 2.0 & 8.0 & 1.19 & 1.34 & 0.72 & 0.58 \\
\hline bh1_N & NFW2 & $6.1 \times 10^{7}$ & $10^{5}$ & $1.0 \times 10^{6}$ & 1 & 2.0 & 4.0 & 2.25 & 2.50 & 0.36 & 0.13 \\
cl1.2_N & NFW2 & $6.1 \times 10^{7}$ & $10^{5}$ & $1.2 \times 10^{6}$ & $10^{4}$ & 2.0 & 4.0 & $\ldots$ & 2.50 & $\ldots$ & $\ldots$ \\
\hline bh15_a & NFW3 & $4.2 \times 10^{8}$ & $10^{5}$ & $1.5 \times 10^{6}$ & 1 & 2.0 & 4.0 & 0.80 & 1.00 & 0.76 & 0.71 \\
bh15_b & NFW3 & $4.2 \times 10^{8}$ & $10^{5}$ & $1.5 \times 10^{6}$ & 1 & 2.0 & 8.0 & 1.25 & 1.70 & 0.61 & 0.44 \\
bh15_c & NFW3 & $4.2 \times 10^{8}$ & $10^{5}$ & $1.5 \times 10^{6}$ & 1 & 2.0 & 12.0 & 2.05 & 3.00 & 0.40 & 0.36 \\
bh15_d & NFW3 & $4.2 \times 10^{8}$ & $10^{5}$ & $1.5 \times 10^{6}$ & 1 & 3.0 & 8.0 & 2.10 & 2.42 & 0.60 & 0.52 \\
cl15_b & NFW3 & $4.2 \times 10^{8}$ & $10^{5}$ & $1.5 \times 10^{6}$ & $10^{4}$ & 2.0 & 8.0 & $\ldots$ & 2.00 & $\ldots$ & $\ldots$ \\
bh10_b & NFW3 & $4.2 \times 10^{8}$ & $10^{5}$ & $1.0 \times 10^{6}$ & 1 & 2.0 & 8.0 & 1.60 & 2.05 & 0.61 & 0.49 \\
\hline bh15_p & NFW3 & $4.2 \times 10^{8}$ & $10^{5}$ & $1.5 \times 10^{6}$ & 1 & 5.0 & 12.0 & $-*$ & 6.00 & 0.25 & 0.15 \\
bh02_a & NFW3 & $4.2 \times 10^{8}$ & $10^{5}$ & $2.0 \times 10^{4}$ & 1 & 2.0 & 4.0 & $-* *$ & 6.00 & 0.75 & 0.75 \\
\hline
\end{tabular}

Note. - NFW1: $V_{c}=30 \mathrm{~km} / \mathrm{s} ; \mathrm{NFW} 2: V_{c}=15 \mathrm{~km} / \mathrm{s}$. NFW1, NFW2: $a=0.1$ and $b=2.5 . \quad \mathrm{NFW} 3: V_{c}=17 \mathrm{~km} / \mathrm{s} ; a=0.5$ and $b=15.0 . \mathrm{t}_{d}$ : time at which the orbit is decayed to a distance from the center $r \leq 30 \mathrm{pc} . \mathrm{t}_{\text {tot }}$ : total time of the simulation. cl1.2_N: $\mathrm{t}_{d}$ cannot be defined as in the above cases since the cluster is no more self-bound at $t \gtrsim 0.8$ Gyr; at $t \sim 1.0$ the remnant is approximately at the center of the host halo. The case of cl15_b is analogous.

* At the end of the simulation the $\mathrm{BH}$ has reached a distance of $\sim 2 \mathrm{kpc}$ from the center of the halo.

* The orbit of the $\mathrm{BH}$ remain stable over the whole duration of the simulations.
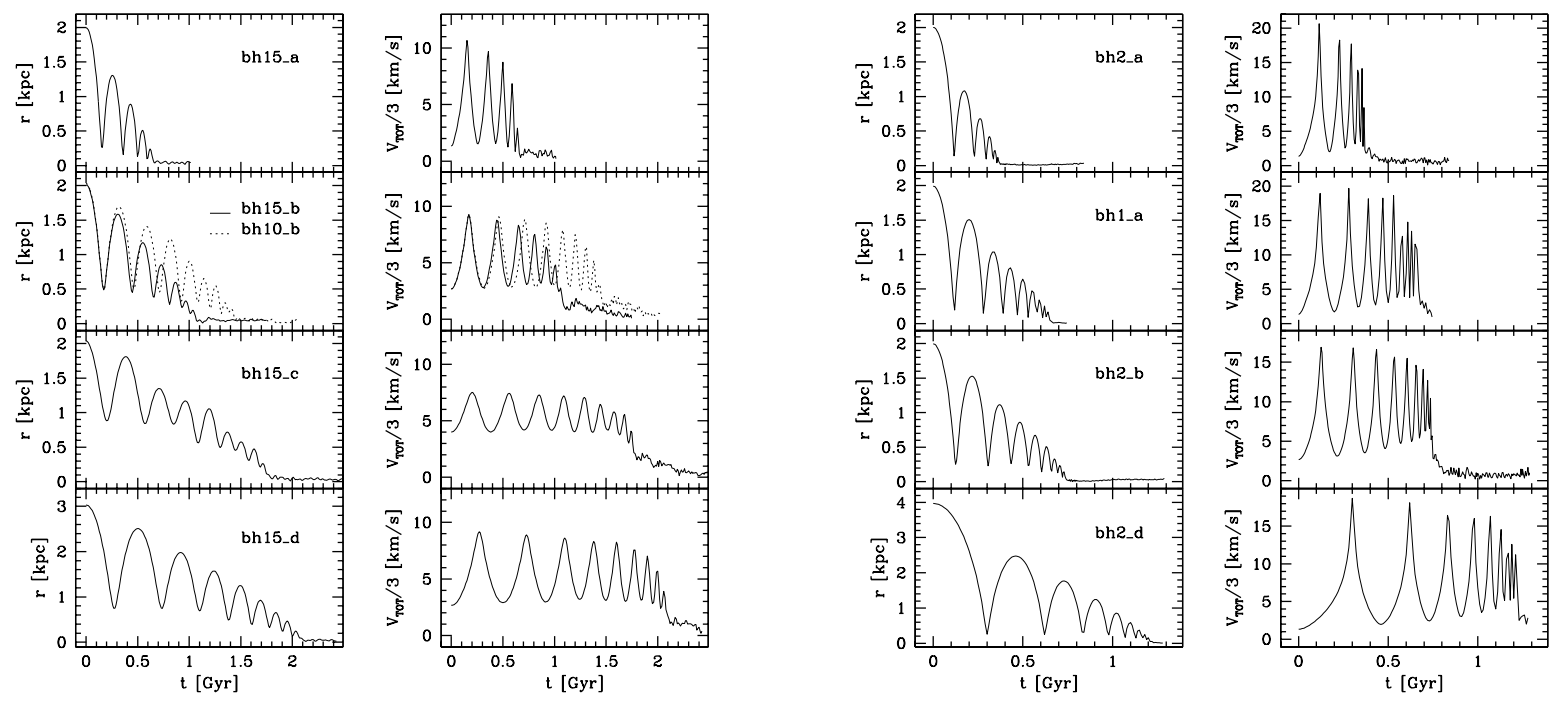

FIG. 19.- Orbital evolution of the single massive particle representing M54 in the N-body experiments described in Tab. 10 Left panels: distance from the center of the host NFW halo as a function of time. Right panels: mean one-component velocity of the massive particle $\frac{V_{T}}{3}$ as a function of time, where $V_{T}=\left(V_{x}^{2}+V_{y}^{2}+V_{z}^{2}\right)^{\frac{1}{2}}$.

$\mathrm{BH}$ as massive as the most massive Sgr cluster (except M54, i.e. Arp $2, M \lesssim 2 . \times 10^{4} M_{\odot}$, adopting the same $(M / L)_{V}$ of $\mathrm{M} 54$, wich seems appropriate given the age and metallicity of the cluster) from a starting point $X_{0}=2.0 \mathrm{kpc}$ and a low initial velocity $\left(V_{Y, 0}=4.0 \mathrm{~km} / \mathrm{s}\right)$. After 6 Gyr no sign of decaying is noticed, the orbit is absolutely stable, in excellent agreement with the results by M05a.

3. The effect of dynamical friction is to progressively decrease the radius, the period and the eccentricity of the orbit. The mean velocity, on the other hand, appears to drop suddenly in the last phases of the decay, possibly when the point-mass particle reaches the dense central cusp of the host NFW halo. This may suggest that the presence of a central cusp may be a crucial ingredient to lead to the observed phase-space coincidence between M54 and Sgr,N, in particular if the recent results by Sánchez-Salchedo. Reves-Iturbide \& Hernandez (2006) and Read et al. (2006) are considered. This suggestion is worth being followed up with specific high-resolution simulations adopting different kinds of host galaxy halo models (such as King 


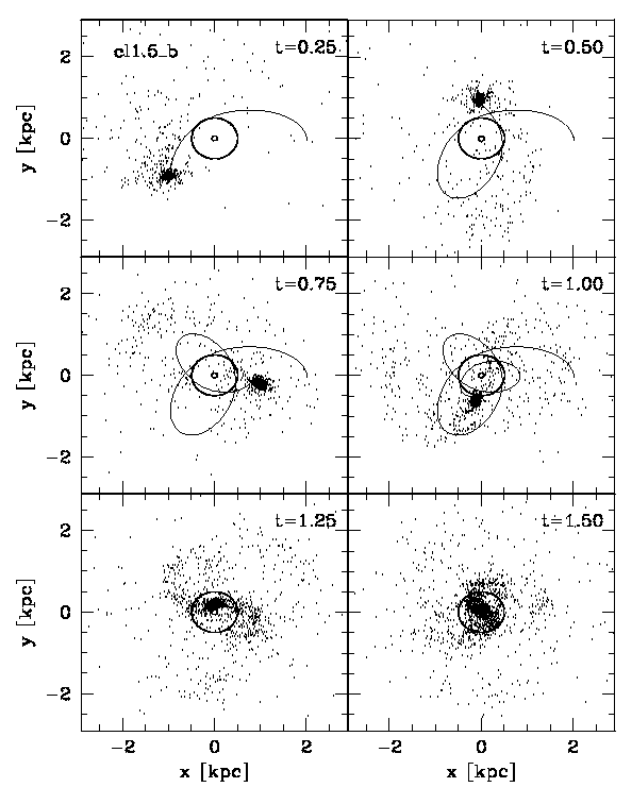

FIG. 20.- Evolution of the M54 cluster model within the NFW model of the Sgr galaxy, for the simulation cl1.2_N (see Tab. 10). In each snapshot of the $\mathrm{x}, \mathrm{y}$ distribution we plot only the points of the M54 model, for clarity. Concentric circles of radius $60 \mathrm{pc}$ and $500 \mathrm{pc}$ are plotted in each panel, for reference. In the top 4 panels we also plotted the orbit of the bh1_N model, to show the approximate path of the center of mass of the cluster model.

models, for instance). Recent studies indicates that dynamical friction is probably much less efficient in cored than in cusped structures (see Sánchez-Salchedo, Reves-Iturbide \& Hernandez 2006; Read et al. 2006; Goerdt et al. 2006).

To check the impact of our adoption of a point-mass model of M54, we repeated the simulation bh1_N and bh15_b adopting a $10^{4}$ particle King model resembling the real cluster as much as possible $\left(W_{0}=8.0, r_{t}=60\right.$ pc; simulations cl1.2_N and cl15_b of Tab. 10); in the case of cl1.2_N the mass was adjusted to $M=1.2 \times$ $10^{6} M_{\odot}$ to have a central l.o.s. velocity dispersion of $\sigma_{0} \simeq$ $15 \mathrm{~km} / \mathrm{s}$. All the final results are fully consistent with those obtained in simulations with a point-mass model for M54, for the purposes of the present study, hence we do not further distinguish between these two classes of models. To illustrate the typical behavior of these live M54 models in Fig. 20 we show a series of snapshots of the evolution of the cluster model of simulation cl15_b. It is interesting to note that in this simulation (as well as in the cl1.2_N one) the cluster suffers strong tidal disruption from the host halo and the final relic sitting nearly at rest at the center of the halo is probably unbound, at odds with the real case. The very fact that the actual cluster is still bound may provide useful constraints on the mass distribution within the Sgr galaxy, that can probably be explored with a systematic suite of N-body simulations.

\section{SUMMARY AND DISCUSSION}

We present the results of a large radial velocity survey of stars in the nucleus of the Sgr galaxy and in the globular cluster M54, which lies at the center of the nucleus itself. From high signal to noise Keck-DEIMOS and VLT-FLAMES spectra, we obtained accurate radial velocities $\left(\epsilon_{V_{r}} \simeq 2 \mathrm{~km} / \mathrm{s}\right)$ and metallicities from the $\mathrm{Ca}$ triplet for 1152 candidate RGB/RC stars of M54/Sgr,N. Selecting by the position in the CMD, by radial velocity and by metallicity, we obtained two reliable and clean samples of $321 \mathrm{Sgr}, \mathrm{N}$ members and 425 M54 stars and we used these samples, further cleaned from $3 \sigma$ velocity outliers, to study the kinematics of the two systems.

In support of this analysis we used archival HST/ACS images to refine the estimates by M05a of the structural parameters of Sgr,N.

We also performed a suite of N-body simulations of the orbital decay (driven by dynamical friction) of a massive cluster into a host dwarf spheroidal galaxy, mimicking the M54 - Sgr system. We focused our attention on the possibility to realize a phase-space match between the infalling cluster and the density cusp of the host NFW halo as close as that observed with M54 and Sgr,N.

The main results of our analyses can be summarized as follows:

1. We have obtained new, much more reliable estimates of the total luminosity and of the typical size of Sgr,N, confirming that it is significantly less luminous than M54 and that it has a different luminosity distribution with respect to the cluster.

2. The systemic radial velocities of M54 and Sgr,N are identical within $\sim \pm 1 \mathrm{~km} / \mathrm{s}$. Coupling this result with those by M05a, it appears that the two systems coincide in phase-space to within the observational errors, at least for what concerns position in space and radial velocity.

3. The velocity dispersion profile of M54 is in good agreement with the theoretical profile of the King model that best fits its surface brightness, at least over a range of 30 core radii $\left(r \leq 3.5^{\prime}\right)$, but possibly also beyond this radius (see Sect. 4.2). In particular, the velocity dispersion drops from $\sigma=14.2$ $\mathrm{km} / \mathrm{s}$ at $r=0$, to $\sigma \simeq 5.3 \mathrm{~km} / \mathrm{s}$ at $r \sim 3.5^{\prime}$. The observed velocity profile strongly suggests that M54 behaves as an ordinary, self-bound globular cluster, at least in its innermost and densest region. In our view, this indicates that even if at the present epoch the motion of its stars is driven by the potential produced by the overall mass distribution within Sgr,N (see below), its velocity profile keeps memory of its original nature, that is an ordinary massive globular cluster orbiting within the Sgr galaxy. A turn-over of the profile to $\sigma=8.8$ $\mathrm{km} / \mathrm{s}$ around the tidal radius of the cluster may suggest that some degree of tidal disruption is occurring in the outskirts of the cluster; however the alternative hypothesis that the sample is contaminated by metal-poor Sgr stars at large radius is also viable and seems more likely, at the present stage (Sect. 4.2).

4. The velocity dispersion profile of $\mathrm{Sgr}, \mathrm{N}$ is consistent with being flat at $\sigma \simeq 10 \mathrm{~km} / \mathrm{s}$ over the whole considered radial range $\left(r \leq 9^{\prime}\right)$. The profile is hardly compatible with any realistic King model roughly reproducing the observed surface brightness profile of Sgr,N. More generally, the fact that the velocity profile is flat in a radial range in which 
the surface density declines by a factor of $>1000$ is strongly uncompatible with any mass-follows-light model (Gilmore et al. 2007). Conversely, a realistic NFW model reasonably reproduces the observed velocity profile and, in principle, does not conflict with the presence of an overdensity of baryons at its center, like Sgr,N. However, it has to be recalled that the NFW model does not make any definite prediction on the SB profile of the embedded stellar nucleus and the compatibility of the observed SB profile of Sgr,N with the NFW model that that fits the velocity profile is not established.

5. M54 and Sgr,N have definitely different kinematical properties. In particular, the velocity dispersion profiles are very different: in the radial range $1.5^{\prime}<$ $r<6.5^{\prime}$ the statistical significance of the difference in the velocity distribution is very large.

6. We have provided observational evidence that the velocity dispersion profile of Sgr remains flat from $r \simeq 1^{\prime}$ to $r \simeq 100^{\prime}$ and that there is no apparent transition in the velocity profile corresponding to the onset of the stellar nucleus, at $r \lesssim 10^{\prime}$. This fact as well as those listed above strongly suggest that Sgr,N and M54 had independent origins, as we would expect that, if the cluster provided the mass seed to collect the Sgr gas that later formed the metal-rich nucleus, Sgr,N stars would have shown a declining velocity dispersion profile, compatible with a mass-follows-light distribution.

7. Our N-body simulations that follow the orbits of massive clusters $\left(1-2 \times 10^{6} M_{\odot}\right.$, representing M54) within different NFW halos (representing the Sgr galaxy) show that for a large range of initial distances and relative velocities, the orbit of the cluster decays completely by dynamical friction within 3 Gyr, at most. Moreover, at the end of the simulations, the cluster is perfectly concentric with the cusp of the host halo (within the resolution of the simulation) and the difference in average velocity is always less than $\sim 2 \mathrm{~km} / \mathrm{s}$. Hence, the observed phase-space coincidence between M54 and Sgr,N can be naturally explained by the "dynamical friction hypothesis" (M05a).

8. According to FC06 the mass of Central Massive Objects, independently if they are Black Holes or stellar nuclei, is $\simeq \frac{3}{1000}$ of the mass of the host galaxy $\left(\frac{M_{C M O}}{M_{\text {gal }}}=0.003\right)$. Using our estimates for the total mass of Sgr,N and M54 we obtain the following estimates for the total mass of the Sgr galaxy, depending on whether we assume is the M54, Sgr,N or the sum of the two (as we would do if we observed the system at the distance of Virgo cluster) is the Central Massive Object: $M_{S g r}=1.0 \times 10^{9} M_{\odot}, M_{S g r}=2.1 \times 10^{9} M_{\odot}$, and $M_{S g r}=3.0 \times 10^{9} M_{\odot}$, respectively. These values are in very good agreement with the independent estimates by Majewski et al. (2003) that ranges from $M_{S g r}=5.8 \times 10^{8} M_{\odot}$ to $M_{S g r}=6.9 \times 10^{9} M_{\odot}$. Hence both Sgr,N and M54 singularly or taken together have a mass compatible with being the CMO of the Sgr galaxy.
9. Both Sgr,N and M54, as well as the combination of the two, when placed in the $M_{V}$ vs. $\log r_{h}$ diagram lie in a region that is populated by globular clusters and galactic stellar nuclei. They are also compatible with the Color-Magnitude relation of nuclei shown by FC06 and with the $M_{V}$ vs. $\sigma$ relation satisfied by globular clusters, nuclei and UCDs (see Geha. Guhathakurta \& van der Marel 2002; Evstigneeva et al. 2007), as shown in Fig. 21 Hence the structure and dynamics of Sgr,N, M54 and their combinations are fully compatible with other galactic nuclei.

10. A detailed study of the mass profile of Sgr,N and M54 using the Schwarzschild method (see Rix et al. 1997; van de Ven et al. 2006, and references therein) is currently ongoing and the results will be presented elsewhere (Ibata et al., in preparation).

These findings lend very strong support to the scenario proposed by M05a to explain the M54/Sgr,N system: the nucleus of the galaxy formed in situ, at the bottom of the potential well of the Sagittarius galaxy; the globular cluster M54 was independently driven to the same site by dynamical friction. As a complement of the above conclusions, it must be recalled that the stellar population that dominates Sgr,N formed several Gyrs later than M54 (see Montegriffo et al. 1998; Saraiedini \& Lavden 1997; Lavden \& Sarajedini 2000; Bellazzini et al. 2006a; Siegel et al. 2007, and references therein). In the present context this does not appear particularly relevant, but it should be kept in mind that M54 can have reached the very center of Sgr after Sgr,N formed its stars (within the last $\sim 5-9$ Gyr Bellazzini et al. 2006a), or even before this, depending on the birthplace of the cluster within the Sgr galaxy. In any case it is very likely that both the cluster and the processed Sgr gas were independently driven - by different mechanisms - to the bottom of the potential well of the Sgr galaxy, i.e. to the center of its Dark Matter halo. A conclusive proof that M54 was driven to its present position by dynamical friction could be provided by the successful detection of genuine extratidal stars at large distances from the cluster center as envisaged in Sect. 4.3, while, as said their non-detection will not disprove the above scenario.

As a possible alternative to this view (or as an extreme version of it) it can be conceived that M54 formed at the bottom of the overall potential well of the Sgr galaxy since the beginning. In this framework M54 and Sgr,N are just the results of two subsequent episodes of star formation both occurring at the very center of Sgr, the second from enriched gas that was infalling on very different orbits with respect to the gas that formed M54, thus resulting in different final stellar kinematics. We regard this possibility as much less likely with respect to the "dynamical-friction" scenario depicted above. First, if M54 is considered as part of the (metal-poor) field population of Sgr, its presence would be at odd with the global metallicity gradient observed by Alard (2001), Bellazzini. Ferraro \& Buonanno (1999), Sarajedini \& Layden (1997) and others in Sgr, as well in all dwarf spheroidal galaxy studied to date (more metal rich and younger populations are preferentially found at 


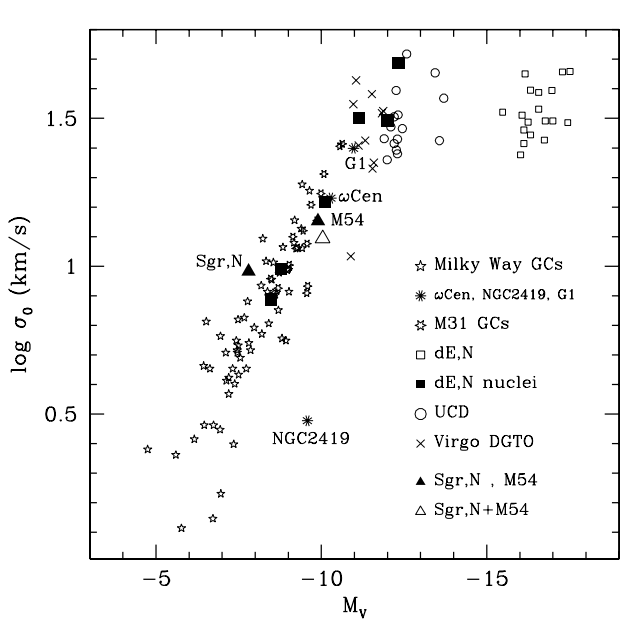

FIG. 21. - Sgr,N and M54 (filled triangles) in the $M_{V}$ vs. $\sigma$ plane (see Evstigneeva et al. 2007). Both systems lie on the locus common to globular clusters, dE nuclei, Dwarf-Globular Transition Objects (DGTO Hasegan et al. 2005), and, possibly, UCDs. The same is true for their combination (Sgr,N+M54, open triangle), i.e. for a system having luminosity equal to the sum of the luminosities of M54 and Sgr,N and having $\sigma$ as estimated from an integrated spectrum obtained with a $1^{\prime \prime}$ slit from the distance of the Virgo cluster of galaxies $(\sigma \simeq 12.4 \mathrm{~km} / \mathrm{s})$. Data for Galactic globulars are from Mackev \& van den Bergh $\left(2005, M_{V}\right)$ and Prvor \& Mevlan (1993, $\sigma$ ); for M31 globulars we took integrated magnitudes from the Revised Bologna Catalog (Galleti et al. 2004) and $\sigma$ from Diorgovski et al. (1997); data for $\mathrm{dE}$ and dE,N are from Geha, Guhathakurta \& van der Marel (2002, 2003); UCDs data are from Drinkwater et al. (2003) and Evstigneeva et al. (2007); data for DGTO are from Hasegan et al. (2005). Some remarkable bright clusters have been labeled, for reference (see Federici et al. 2007).

the center of dSph's Harbeck et al. 2001). While dE nuclei have been indicated as a possible exceptions to this trend, the ingestion of large metal poor globular clusters in pre-existing metal rich nuclei seems one possible natural way to reconcile the generally observed metallicity gradients and the presence of nuclei that are bluer than their parent galaxy (Lotz, Miller \& Ferguson 2004). Second, as both M54 and Sgr,N would have formed from gas falling into the same potential well, the reason for the different kinematics remains to be explaned, while it is a natural outcome if M54 formed elsewhere as a classical globular cluster.

\subsection{The process of galaxy nucleation}

If we take for demonstrated the above concluding remarks, we can ask what we have learned about the process of galaxy nucleation from the case studied here. Concerning the two main mechanisms that have been put forward in the literature, i.e. (a) formation of the nucleus by infall of globular cluster(s) to the center of the galaxy, or (b) in situ formation by accumulation of gas at the center of the potential well and its subsequent conversion into a stellar overdensity (see Sect. 1. and Grant, Kuipers, \& Phillipps 2005; Côté et al. 2006), the main conclusion that can be drawn from the case of Sgr is that both channels are viable and actually both have been at work "simultaneously" in Sgr.

The present analysis has shown that a stellar nucleus made of the typical material that dominates the baryonic mass budget of Sgr is present in this galaxy, independent of M54, as it display the same flat velocity dispersion profile as the whole core of Sgr, much different from that of the cluster.

In a likely scenario, the enriched gas from previous generations of Sgr stars accumulated at the bottom of the overall potential well of the galaxy, until star formation transformed it into a stellar nucleus whose surface brightness is $\gtrsim 100$ times larger than in the surrounding Sgr core, a substructure within a larger galaxy. On the other hand, M54 is a (relatively) ordinary massive, old and metal poor globular cluster. Independent of its birthplace within the early Sgr galaxy, its mass and the density of the surrounding medium of the host galaxy drove it to the bottom of the Sgr potential well, by dynamical friction. During its trip to the densest central region of Sgr, the dense cluster managed to survive the tidal force of the host galaxy, hence it reached the present position as a (partially?) self-bound stellar system.

While the mass budget at the center of Sgr is probably dominated by the underlying DM halo, M54 dominates the overall light distribution : an observer taking photometry and/or spectra of the unresolved nucleus of Sgr from a distant galaxy (say, a galaxy in the Virgo cluster) would find that the object looks like a bright and blue globular cluster; the integrated velocity dispersion would not show any peculiar feature revealing the composite nature of the observed nucleus (see Fig. 21, above). Probably, it would be impossible to disentangle the two systems from the integrated light ${ }^{10}$.

Finally, the Sgr case seems to support the observed ubiquity of nuclei (see Sect. 1. and C06). The Sgr galaxy was able to form a sizable nucleus "twice" and with two different formation channels: if either of the two channels had not been viable for some reason, the galaxy would have ended up with a nucleus in any case. The fact that Sgr is the only case of galaxy with a clear nucleus among those classified as dwarf spheroidals in the Local Group may suggest that the progenitor of Sgr was in fact a brighter $\mathrm{dE}$ or disc galaxy that has been transformed into a dSph by the interaction with the Milky Way (Majewski et al. 2003; Mayer et al. 2007, M05b, and references therein)

\subsection{Suggestions for further investigations}

The results presented in this paper suggest several interesting lines of research that we did not follow up for practical reasons. However we feel that it is worth briefly mentioning some of them here, as a possible starting point for future studies.

- The results presented by Read et al. (2006), Goerdt et al. (2006) and Sánchez-Salchedo, Reves-Iturbide \& Hernandez (2006), suggest that the complete decay of a massive cluster to the very center of the host galaxy is much easier and faster if a central density cusp is present. It is even possible that a central cusp is actually required to bring a

${ }^{10}$ It would be interesting to check if there is some spectral feature that may reveal the composite nature of the "system", in the present case. We plan to do this in the future by combining properly scaled synthetic spectra representing the light output of M54 and Sgr,N. 
cluster down to the very bottom of the overall potential well. If this is the case, the position of M54 within Sgr,N would support the existence of a central cusp in actual DM halos, a point that has been questioned by several authors (see Sánchez-Salchedo, Reves-Iturbide \& Hernandez 2006, and references therein). The "complete infall" of a massive cluster may need a NFW cusp and, simultaneously, it may transform the cusp into a core by transferring orbital energy and momentum to the surrounding "medium", thus possibly providing a self-regulating mechanism that simultaneously prevent the further decay of other clusters (that, in general cases, would be quite difficult, given the expected decay times, see Hernandez \& Gilmore 1998; Oh, Lin \& Richer 2000; Read et al. 2006; Sánchez-Salchedo. Reves-Iturbide \& Hernandez 2006; Goerdt et al. 2006, and references therein). In this context, it may be worth to recall also the work by Strigari et al. (2006), whose results militates against the presence of a large-size core in the Fornax dSph, and by Boylan-Kolchin \& Ma (2004) on the resilience of cuspy halos in major mergers. These ideas seems worthy of detailed theoretical follow up.

- It has been suggested several times that bright and metal poor globular clusters may be of cosmological origin (see Brodie \& Strader 2006; Maschenko \& Sills 2005, and references therein). If this is the case they may be very intimately linked to the earliest phases of formation of galaxies and there may have been plenty of opportunities for most of them to become the nuclei of some dwarf galaxy. The tidal stress that they suffered during their infall to the center of their host galaxies may be at the origin of the larger half-light sizes that are observed in those of them that have been suggested as possible nuclear remnants of ancient dwarf galaxies (Freeman \& Bland-Hawthorn 2002; Federici et al. 2007; Mackey \& van den Bergh 2005). This kind of scenario may be explored with dedicated N-body simulations, possibly including gas and stars. It would be interesting also to consider in detail the results presented here in relation with the scenario for the origin of globular clusters recently discussed by Böker (2007).

- All the analysis presented in this paper have been performed within the standard Newtonian gravitation theory and dynamics. It may be worth to consider the observational scenario emerged from this study also in the framework of Modified Newtonian Dynamics paradigms (MOND, see Milgrom 2008; Sanders \& McGaugh 2002, and references therein), even if it may not necessarily be an ideal case. The transition between ordinary Newtonian regime and MOND regime occurs around $r \simeq 4.5^{\prime}-6.5^{\prime}$, depending on the actual stellar mass of $\mathrm{M} 54+\mathrm{Sgr}, \mathrm{N}$, that is in a range covered by our data.

- There is little doubt that the final fate of the Sgr galaxy will be its complete tidal disruption. Once the large scale stellar body of Sgr will be completely dispersed into the Galactic halo, the final remnant of this (once) relatively large galaxy would be a faint nucleus embedding a bright globular cluster. An observer lacking any knowledge of the origin of this object would conclude that it is a very bright and peculiar globular cluster, dominated by a metal poor population $([\mathrm{Fe} / \mathrm{H}] \sim-1.5$, possibly with some spread) but also including a small fraction $(\sim 10 \%)$ of metal rich stars (with average $[\mathrm{Fe} / \mathrm{H}] \sim-0.4)$. Also the abundance patterns would appear different: for instance, the metal poor (M54) stars would appear as moderately $\alpha$ enhanced (Brown. Wallerstein \& Gonzalez 1999), while metal rich stars (Sgr,N) would have solar or sub-solar $[\alpha / F e]$ ratios (Monaco et al. 2005b). The radial velocities would reveal that the metal rich and metal poor stars have the same systemic velocity, but different velocity dispersion profiles and slightly different density profiles, possibly with some rotation in the metal poor component. However the dominance of metal-poor (M54) stars would be so high that there would be no hint of the presence of a Dark Matter component. The half-light radius of the system would appear slightly larger with respect to ordinary globulars (Mackey \& van den Bergh 2005; Federici et al. 2007). Most of these likely characteristics of the future remnant of Sgr seem to have a counterpart in the widely studied and mysterious stellar system $\omega$ Centauri (see Norris et al. 1997; van Leeuwen. Huges \& Piotto 2002; Pancino et al. 2000, 2002, 2003; van de Ven et al. 2006, and references therein), that has been proposed as the possible remnant of a nucleated dwarf elliptical since Freeman (1993). While there are also noticeable differences between the two cases, the analogy seems very intriguing and potentially powerful ${ }^{11}$. It is possible that at least some of the observational features of $\omega$ Centauri that appear so difficult to explain may find their natural place within a scenario like the one described above.

M.B. acknowledges the financial support of INAF through the Grant PRIN05 CRA 1.06.08.02. M.B. is grateful to the Observatoire de Strasbourg for the kind hospitality during the period in which most of the N-body simulations presented here have been performed. This work is partially based on observations made with the European Southern Observatory telescopes (WFI@2.2m) as part of the observing program 65.L-0463. Partially based on observations made with the ESO Very Large Telescope (VLT/FLAMES) as part of the observing program 075.D-0075. Partially based on Advanced Camera for Surveys (ACS) observations col-

11 It is interesting to recall that $\omega$ Cen shares with Sgr some remarkable chemical peculiarities. In particular, stars of comparable metallicity in the two systems are strongly enhanced in s-process elements and the ratio of heavy s-process to light s-process elements [hs/ls] is very similar. Furthermore, $\omega$ Cen and Sgr are, up to now, the only stellar systems known to have deficient $[\mathrm{Cu} / \mathrm{Fe}]$ ratios with respect to the trend in the Galactic disk and halo (McWilliam \& Smecker-Hane 2005) 
lected with the Hubble Space Telescope within the programme GO 10755. This research has made use of the NASA Astrophysics Data System Abstract Service.
Facilities: Keck (DEIMOS), ESO-VLT (FLAMES), MPG-ESO (WFI@2.2m), HST (ACS).

\section{REFERENCES}

Alard, C., 2001, A\&A, 377, 389

Armandroff, T.E., 1989, AJ, 97, 375

Balcells, M., Graham, A.W., \& Peletier, R.F., 2007, ApJ, 665, 1084

Bassino, L.P., Muzzio, J.C., \& Rabolli, M., ApJ, 431, 634

Bassino, L.P., \& Muzzio, J.C., 1995, The Observatory, 115, 256

Battaglia, G., Irwin, M., Tolstoy, E., Hill, V., Helmi, A., Letarte, B., \& Jablonka, P., 2008, MNRAS, 383, 183

Bekki, K., Couch, W.J., Drinkwater, M.J., \& Shioya, Y., 2004, ApJ, 610, L13

Bellazzini, M., Ferraro, F.R., \& Buonanno, R., 1999, MNRAS, 307, 619

Bellazzini, M., Ferraro, F.R., \& Ibata, R.A., 2003a, AJ, 125, 188

Bellazzini, M., Ibata, R.A., Ferraro, F.R., \& Testa, V., 2003b, A\&A, 405, 577

Bellazzini, M., Correnti, M., Ferraro, F.R., Monaco, L., \& Montegriffo, P., 2006a, A\&A, 446, L1

Bellazzini, M., Newberg, H.J., Correnti, M., Ferraro, F.R., Monaco, L., \& Montegriffo, P., 2006b, A\&A, 457, L21

Binggeli, B., Sandage, A., \& Tammann, G.A., 1985, AJ, 90, 1681

Binggeli, B., Tammann, G.A., \& Sandage, A., 1987, AJ, 94, 251

Böker, T., Laine, S., van der Marel, R.P., Sarzi, M., Rix, H.-W., Ho, L., \& Shields, J.C., 2004, AJ, 127, 105

Böker, T., 2007, ApJ, in press (arXiv:0711.4542)

Bonifacio, P., et al., 2006, in Chemical Abundances and Mixing in Stars in the Milky Wai and its Satellites, S. Randich \& L. Pasquini Eds., ESO Astrophysics Symposia, Berlin, Springer, p. 232

Boylan-Kolchin, M., \& Ma, C.-P., 2004, MNRAS, 349, 1117

Brandt, S., 1970, Statistical and Computational Methods in Data Analysis, North-Holland Publishing Co., Amsterdam/London

Brodie, J.P., \& Strader, J., 2006, ARA\&A, 44, 193

Brown, J.A., Wallerstein, G., Gonzalez, G., 1999, AJ, 118, 1245

Burton, W. B., \& Lockman, F. J. 1999, A\&A, 349, 7

Carollo, C. M., Stiavelli, M., \& Mack, J., 1998, AJ, 116, 68

Carraro G., Zinn, R., \& Moni Bidin, C., 2007, A\&A, 466, 181

Carrera, R., Gallart, C., Pancino, E., Zinn, R., 2007, AJ, 134, 1298

Carretta, E., Gratton, R. 1997, A\&AS 121, 95

Catelan, M., in XXI Century Challenges for Stellar Evolution, S. Cassisi \& M. Salaris, Mem. SAIt, 79, in press (arXiv:0711.2761) Chandrasekhar, S., 1943, ApJ, 97, 255

Colpi, M., Mayer, L. \& Governato, F., 1999, ApJ, 525, 720

Cordier, D., Pietrinferni, A., Cassisi, S., \& Salaris, M., 2007, AJ, 133,468

Côté, P., et al., 2004, ApJS, 153, 223

Côté, P., et al., 2006, ApJS, 165, 57 (C06)

Da Costa, G.S., \& Armandroff, T.E., 1995, AJ, 109, 2533

Davis, M., et al. 2003, Proc. SPIE, 4834, 161

Dehnen, W., Binney, J. 1998, MNRAS, 294, 429

Dehnen, W., 2000, ApJ,536, L39

Dehnen, W., 2002, J. Comput. Phys., 179, 27

Dinescu, D.I, Girard, T.M., van Altena, W.F., \& López, C.E., 2005, ApJ, 618, L25

Djorgovski, S.G., Gal., R.R., McCarthy, J.K., de Carvalho, R.R., Meylan, G., Bendinelli, O., \& Parmeggiani, G., 1997, ApJ, 474, L19

Drinkwater, N.J., Gregg, M.D., Hilker, M., Bekki, K., Couch, W.J., Ferguson, H.C., Jones, J.B., \& Phillipps, S., 2003, Nature, 423, 519

Evstigneeva, E.A., Gregg, M.D., Drinkwater, M.J., \& Hilker, M., 2007, AJ, 133, 1722

Evans, N.W., Wilkinson, M.I., Perrett, K.M., Bridges, T.J., 2003, ApJ, 583, 752

Federici, L., Bellazzini, M., Galleti, S., Fusi Pecci, F., Buzzoni, A., \& Parmeggiani, G., 2007, A\&A, 473, 429

Ferguson, H.C., \& Binggeli, B., 1994, A\&A Rev., 6, 67

Ferrarese, L. et al., 2006, ApJS, 164, 334

Ferrarese, L., \& Côté, P., 2006, in Black Holes: from Stars to Galaxies - across the Range of Masses, V. Karas and G. Matt Eds., Cambridge, UK: Cambridge University Press, IAU Symp. 238,261 (FC06)
Freeman, K.C., 1993, in The Globular Cluster - Galaxy Connection, G.H. Smith and J.P. Brodie Eds., ASP Conf. Ser., 48, 608

Freeman, K.C., \& Bland-Hawthorn, J., 2002, ARA\&A, 40, 487

Fujii, M., Funato, Y., \& Makino, J., 2006, PASJ, 58, 743

Gallagher, J.S., \& Wyse, R.F.G., 1994, PASP, 106,1225

Galleti, S., Federici, L., Bellazzini, M., Fusi Pecci, F., \& Macrina, S., 2004, A\&A, 416, 917

Geha, M., Guhathakurta, P., \& van der Marel, R.P., 2002, AJ, 124, 3073

Geha, M., Guhathakurta, P., \& van der Marel, R.P., 2003, AJ, 126, 1794

Gilmore, G., Wilkinson, M.I., Wyse, R.F.G., Kleyna, J.T., Koch, A., Wyn Evans, N., Grebel, E.K., 2007, ApJ, 663, 948

Goerdt, T., Moore, B., Read, J. I., Stadel, J., \& Zemp, M. 2006 , MNRAS, 368, 1073

Graham, A.W., \& Guzmán, R., 2003, AJ, 125, 2936

Grant, N.I., Kuipers, J.A., \& Phillipps, S., 2005, MNRAS, 363, 1019

Haller, J.W., \& Melia, F., 1996, ApJ, 464, 774

Harbeck, D., Grebel, E.K., Holtzman, J., Guhathakurta, P., Brandner, W., Geisler, D., Sarajedini, A., Dolphin, A., HurleyKeller, D., \& Mateo, M., 2001, AJ, 3092

Harris, W.E, 1996, AJ, 112, 1487

Hasegan, M., et al., 2005, ApJ, 627, 203

Hargreaves, J. C., Gilmore, G., \& Annan, J. D. 1996, MNRAS, 279,108

Heisler, J., Tremaine, S., \& Bachall, J.N., 1985, ApJ, 298, 8

Hernandez, X., Gilmore, G., 1998, MNRAS, 297, 517

Holtzman, J., Afonso, C., \& Dolphin, A., 2006, ApJS, 166, 534.

Ibata, R.A., Irwin M.J., \& Gilmore G., 1994, Nature

Ibata, R.A., Wyse, R.F.G., Gilmore, G., Irwin, M.J., Suntzeff, N.B., 1997, AJ, 113, 634

Ibata, R., Lewis, G. 1998, ApJ 500, 575

Ibata R., Lewis G., Irwin M., Totten E. \& Quinn T., 2001, ApJ, 551,294

Ibata, R., Chapman, S., Ferguson, A., Lewis, G., Irwin, M., Tanvir, N., 2005, ApJ 634, 287

Illingworth, G., 1976, ApJ, 204, 73

Illingworth, G., \& Illingworth, W., 1976, ApJS, 30, 227

King, I.R., 1962, AJ, 67, 471

King, I.R., 1966, AJ, 71, 64

Layden, A.C. \& Sarajedini, A., 2000, AJ, 119, 1760 (LS00)

Lotz, J.M., Telford, R., Ferguson, H.C., Miller, B.W., Stiavelli, M., \& Mack, J., 2001, ApJ, 572

Lotz, J.M., Miller, B.W., \& Ferguson, H.C., 2004, ApJ, 613, 262

Lupton, R., 1993, Statistics in theory and practice, Princeton, Princeton University Press

Mackey, A.D., \& Gilmore, G.F., 2003, MNRAS, 340, 175

Mackey, A.D., \& van den Bergh, S., 2005, MNRAS, 360, 631

Majewski, S.R., Skrutskie, M.F., Weinberg, M.D., Ostheimer, J.C., 2003, ApJ, 599, 1082

Mandushev, G., Staneva, A.. \& Spasova, N., 1991, A\&A, 252, 94

Martin, N.F., Ibata, R.A., Chapman, S.C., Irwin, M.J., \& Lewis, G.F., 2007, MNRAS, 380, 281

Maschenko, S., \& Sills, A., 2005, ApJ, 619, 243

Mayer, L., Kzantzidis, S., Mastropiero, C., \& Waldsley, J., Nature, 445, 738

McLaughlin, D.E., van der Marel, R.P., 2005, ApJS, 161, 304

McWilliam, A., \& Smecker-Hane, T., 2005, ApJ, 622, L29

Mieske, S., et al., 2006, ApJ, 653, 193

Mieske, S., \& Baumgardt, H., 2007, A\&A, 475, 851

Milgrom, M., 2008, in Matter and energy in the Universe: from nucleosynthesis to cosmology, XIX Rencontres de Blois, in press (arXiv:0801.3133)

Miocchi, P., Capuzzo Dolcetta, R., Di Matteo, P., \& Vicari, A., 2006, ApJ, 644, 940

Monaco, L., Ferraro, F.R., Bellazzini, M., \& Pancino, E., 2002, ApJ, 578, L47

Monaco, L., Bellazzini, M., Ferraro, F.R., \& Pancino, E., 2003, ApJ, 597, L25 
Monaco, L., Bellazzini, M., Ferraro, F.R., \& Pancino, E., 2003, ApJ, 597, L25

Monaco, L., Bellazzini, M., Ferraro, F.R., \& Pancino, E., 2005a, MNRAS, 356, 1396 (M05a)

Monaco, L., Bellazzini, M., Bonifacio, P., Ferraro, F.R., Marconi, G., Pancino, E., Sbordone, L., \& Zaggia, S., 2005b, A\&A, 441, 141 (M05b)

Monaco, L., Bellazzini, M., Bonifacio, P., Buzzoni, A., Ferraro, F.R., Marconi, G., Sbordone, L., \& Zaggia, S., 2007, A\&A, 464, 201

Montegriffo, P., Bellazzini, M., Ferraro, F.R., Martins, D., Sarajedini, A., Fusi Pecci, F., 1998, MNRAS, 294, 315

Muñoz, R.R., Majewski, S.R., \& Johnston, K.V., 2007, ApJ, submitted (arXiv:0712.4312)

Navarro, J.F., Frenk, C.S., \& White, S.D.M., 1996, ApJ, 462, 563 (NFW)

Norris, J.E., Freeman, K.C., Mayor, M., \& Seitzer, P., 1997, ApJ, 487, L187

Noyola, E., \& Gebhardt, K., 2006, AJ, 132, 447

Oh, K.S., Lin, D.N.C., \& Richer, H.B., 2000, ApJ, 531, 727

Olszewski, E. W., Pryor, C., \& Armandroff, T. E. 1996, AJ, 111, 750

Pancino, E., Ferraro, F.R., Bellazzini, M., Piotto, G., \& Zoccali, M., 2000, ApJ, 534, L83

Pancino, E., Pasquini, L., Hill, V., Ferraro, F.R., \& Bellazzini, M., 2002, ApJ, 568, L101

Pancino, E., Seleznev, A., Ferraro, F. R., Bellazzini, M., Piotto, G., 2003, MNRAS, 345, 683

Peterson, C.J., 1993, in Structure and Dynamics of Globular Clusters, S. Djorgovsky \& G. Meylan Eds., ASP Conf. Ser., 50, 337

Pryor, C., \& Meylan, G., 1993, in Structure and Dynamics of Globular Clusters, S. Djorgovsky \& G. Meylan Eds., ASP Conf. Ser., 50, 357

Read, J.I., Goerdt, T., Moore, B., Pontzen, A.P., Stadel, J., Lake, G., 2006, MNRAS, 373, 1451

Renzini, A., \& Buzzoni, A., 1986, in Spectral evolution of galaxies, Dordrecht, D. Reidel Publishing Co., p. 195

Renzini, A., \& Fusi Pecci, F., 1988, ARA\&A, 26, 199
Rix, H.-W., de Zeeuw, P.T., Cretton, N., van der Marel, R.P., \& Carollo, M., 1997, AJ, 488, 702

Robin, A.C., Reylé, C., Derrière, S., Picaud, S., 2003, A\&A, 409, 523

Rosenberg, A., Recio-Blanco, A., \& Garcìa-Marìn, M., 2004, ApJ, 603, 135

Rossa, J., van der Marel, R.P., Böker, T., Gerssen, J., Ho, L.C., Rix, H.-W., Shields, J.C., \& Walcher, C.-J., 2006, ApJ, 132, 1074 Rutledge, G.A., Hesser, J.E., \& Stetson, P.B., 1997, PASP, 109, 907

Sánchez-Salchedo, F.J., Reyes-Iturbide, J., \& Hernandez, X., 2006, MNRAS, 370, 1829

Sanders, R.H., \& McGaugh, S.S., 2002, ARA\&A, 40, 263

Sarajedini, A., Layden, A.C., 1995, AJ, 109, 1086

Sarajedini, A., Layden, A.C., 1997, AJ, 113, 264

Sarajedini, A., et al., 2007, AJ, 133, 1658

Siegel, M.H., et al., 2007, ApJ, 667, L57

Smith, E.O., Rich, R.M., \& Neill, J.D., 1998, AJ, 115, 2369

Sollima, A., Beccari, G., Ferraro, F.R., Fusi Pecci, F., \& Sarajedini, A., 2007, MNRAS, 380, 781

Strigari, L.E., Bullock, J.S., Kaplinghat, M., Kravtsov, A.V., Gnedin, O.Y., Abazajian, K., Klypin, A.A., 2006, ApJ, 652, 306

Teuben, P.J., 1995, in Astronomical Data Analysis Software and Systems IV, R. Shaw, H.E. Payne and J.J. Hayes Eds., ASP Conf. Ser., 77, 398

Trager, S.C., King, I.R., \& Djorgovski, S.G., 1995, AJ, 109, 218

van Leeuwen, F., Hughes, J.D., \& Piotto, G., 2002, $\omega$ Centauri, A Unique Window into Astrophysics, ASP Conf. Ser., vol. 265

Tremaine, S.D., Ostriker, J.P., \& Spitzer, L.Jr., 1975, ApJ, 196, 407

Valluri, M., Ferrarese, L., Meritt, D., \& Joseph, C.L., 2005, ApJ, 628,137

van de Ven, G., van den Bosch, R.C.E., Verolme, E.K., de Zeeuw, P.T., 2006, A\&A, 445, 513

Walker, M.G., Mateo, M., Olszewki, E.W., Bernstein, R., Wang, X., \& Woodroofe, M., 2006, AJ, 131, 2114

Webbink, R.F., 1985, in Dynamics of star clusters, J. Goodman \&

P. Hut Eds., Kluwer, Dordrecht, IAU Symp. 113, 541

Wehner, E.H., \& Harris, W.E., 2006, ApJ, 644, L17 